\title{
Enantioselective Transfer Aminoallylation: Synthesis of Optically Active Homoallylic Primary Amines
}

\author{
Masaharu Sugiura, Chieko Mori and Shū Kobayashi* \\ Graduate School of Pharmaceutical Sciences, The University of Tokyo \\ Hongo, Bunkyo-ku, Tokyo 113-0033, Japan
}

\section{Supporting Information}

Table of Contents

Experimental Details and Physical Data

General Methods

Chemicals

Preparation of chiral amine 1a

Assignment of the relative configuration of $\mathbf{1 a}$

Transfer aminoallylation of glyoxylic acid (2a)

General procedures for transfer aminoallylation using 1a (Tables 1 and 2)

Determination of the enantiomeric excess of $(S)$-citronellal

Isolation of intermediate $\mathbf{4 b}$

Isolation of intermediate $\mathbf{5 b}$

$\mathrm{NMR}$ analysis of reaction of $\mathbf{4 b}$ in $\mathrm{CDCl}_{3}$

$\mathrm{NMR}$ analysis of reaction of $\mathbf{5 b}$ in $\mathrm{CDCl}_{3}$

NMR analysis of reaction of 2-thiophenecarboxaldehyde (2f) with $\mathbf{1 a}$ in $\mathrm{CDCl}_{3} \quad \mathrm{~S}-16$

References

S-17

NMR spectra for all new compounds

\section{Experimental Details and Physical Data}

General Methods: ${ }^{1} \mathrm{H}$ and ${ }^{13} \mathrm{C}$ NMR spectra were recorded on JEOL JNM-ECX400 or JNMECX600 spectrometer in $\mathrm{CDCl}_{3}$ unless otherwise noted. Tetramethylsilane (TMS) served as an internal standard $(\delta=0)$ for ${ }^{1} \mathrm{H}$ NMR and $\mathrm{CDCl}_{3}$ was used as an internal standard $(\delta=$ 77.0) for ${ }^{13} \mathrm{C}$ NMR. Electrospray ionization high-resolution mass spectra (ESI-HRMS) were measured with BRUKER DALTONICS BioTOF II mass spectrometer. Optical rotations 
were recorded on JASCO P-1010 polarimeter. Preparative thin-layer chromatography (PTLC) was carried out using Wakogel B-5F. Ethanol was distilled from sodium and stored over $3 \AA$ MS. 1,2-Dichloroethane (DCE) was distilled from $\mathrm{P}_{2} \mathrm{O}_{5}$ and then from $\mathrm{CaH}_{2}$ and stored over $4 \AA$ MS. Purified water was obtained by MILLIPORE water purification system. All other solvents were purified based on standard procedures.

Chemicals: (1R)-(-)-Camphorquinone was purchased from Tokyo Kasei Kogyo Co., Ltd. and used without further purification. Allylboronoic acid pinacol ester was prepared according to the literature. ${ }^{1}$ Methanolic ammonia (ca. $7 \mathrm{M}$ ) was purchased from Aldrich Chemical Company. Glyoxylic acid monohydrate (2a) was purchased from Wako Pure Chemical Industries, Ltd. and used without further purification. Aldehydes $\mathbf{2 b} \mathbf{b} \mathbf{i}, \mathbf{2 k}$, and $\mathbf{2 m}$ were purchased from Tokyo Kasei Kogyo Co., Ltd. and freshly distilled or recrystallized (for 2c and 2d) before use. Aldehydes 2j, 2l, and $\mathbf{2 0}$ were purchased from Aldrich Chemical Company and freshly distilled prior to use. Aldehyde 2n was prepared by Swern oxidation of 3-benzyloxy-1-propanol. All other chemicals were purified based on standard procedures.

\section{Preparation of $(1 R, 3 R, 4 S)$-3-allyl-3-amino-1,7,7-trimethylbicyclo[2.2.1]heptan-2-one (1a)}

To a solution of $(1 R)-(-)$-camphorquinone $(1.33 \mathrm{~g}, 8.0 \mathrm{mmol})$ in methanolic ammonia (ca. 7 M, $12 \mathrm{~mL})$, allylboronoic acid pinacol ester (2.02 g, $12.0 \mathrm{mmol})$ was added dropwise at $\mathrm{rt}$. After being stirred for $24 \mathrm{~h}$ at that temperature, $3 \mathrm{~N}$ hydrochloric acid was added slowly to adjust $\mathrm{pH}$ ca. 1. After being stirred for $30 \mathrm{~min}$, the aqueous layer was washed with dichloromethane $(3 \times)$, basified with $6 \mathrm{~N}$ aqueous $\mathrm{NaOH}(\mathrm{pH}$ ca. 10), and extracted with dichloromethane $(3 \times)$. The later dichloromethane layers were dried over anhydrous $\mathrm{Na}_{2} \mathrm{CO}_{3}$, filtered, evaporated, and purified by silica gel column chromatography (hexane/ethyl acetate $=3 / 1)$ to give amine $1 \mathbf{a}(1.32 \mathrm{~g}, 80 \%$ yield, $>99 \% \mathrm{de})$.

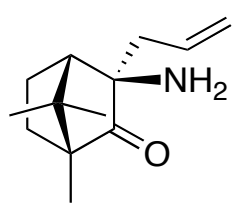

\section{$(1 R, 3 R, 4 S)$-3-Allyl-3-amino-1,7,7-trimethylbicyclo[2.2.1]heptan-2-one (1a)}

$[\alpha]_{\mathrm{D}}^{23}+151.6\left(\mathrm{c} 1.26, \mathrm{CHCl}_{3},>99 \% \mathrm{de}\right) ;{ }^{1} \mathrm{H} \mathrm{NMR}\left(400 \mathrm{MHz}, \mathrm{CDCl}_{3}\right.$ ) $\delta 5.85$ (dddd, $J=16.9$, 10.3, 8.0, 6.4 Hz, 1H), 5.22-5.12 (m, 2H), $2.25(\mathrm{dd}, J=14.6,6.2 \mathrm{~Hz}, 1 \mathrm{H}), 2.11(\mathrm{dd}, J=14.6$, $7.8 \mathrm{~Hz}, 1 \mathrm{H}), 1.94-1.83(\mathrm{~m}, 1 \mathrm{H}), 1.85(\mathrm{~d}, J=3.2 \mathrm{~Hz}, 1 \mathrm{H}), 1.72-1.62(\mathrm{~m}, 2 \mathrm{H}), 1.52-1.44(\mathrm{~m}$, 
1H), 1.41 (brs, 2H), 1.07 (s, 3H), 1.00 (s, 3H), 0.91 (s, 3H); $\left.{ }^{13} \mathrm{C} \mathrm{NMR} \mathrm{(100} \mathrm{MHz,} \mathrm{CDCl}_{3}\right) \delta$ 222.8, 133.3, 119.3, 61.9, 58.6, 52.5, 46.3, 42.5, 30.2, 23.3, 23.0, 20.9, 9.8; ESI-HRMS calcd for $\mathrm{C}_{13} \mathrm{H}_{22} \mathrm{NO}\left(\mathrm{M}+\mathrm{H}^{+}\right)$208.1696, found 208.1689.

\section{Assignment of the relative configuration of $1 \mathrm{a}$}

A mixture of $1 \mathrm{a}$ (29.5 mg, $0.14 \mathrm{mmol})$, triethylamine (1.6 equiv), DMAP (0.05 equiv), and $p$ bromobenzoyl chloride (37.0 mg, 1.2 equiv) in dichloromethane (1.4 mL) was stirred at $\mathrm{rt}$ for $24 \mathrm{~h}$. The mixture was concentrated and directly purified by preparative TLC (hexane/ethyl acetate $=5 / 1)$ to give the corresponding $p$-bromobenzamide $(36.7 \mathrm{mg}, 66 \%$ yield $)$. A NOESY experiment of this $p$-bromobenzamide confirmed the $3 R$ configuration (Chart S- 1 and Figure S-1).

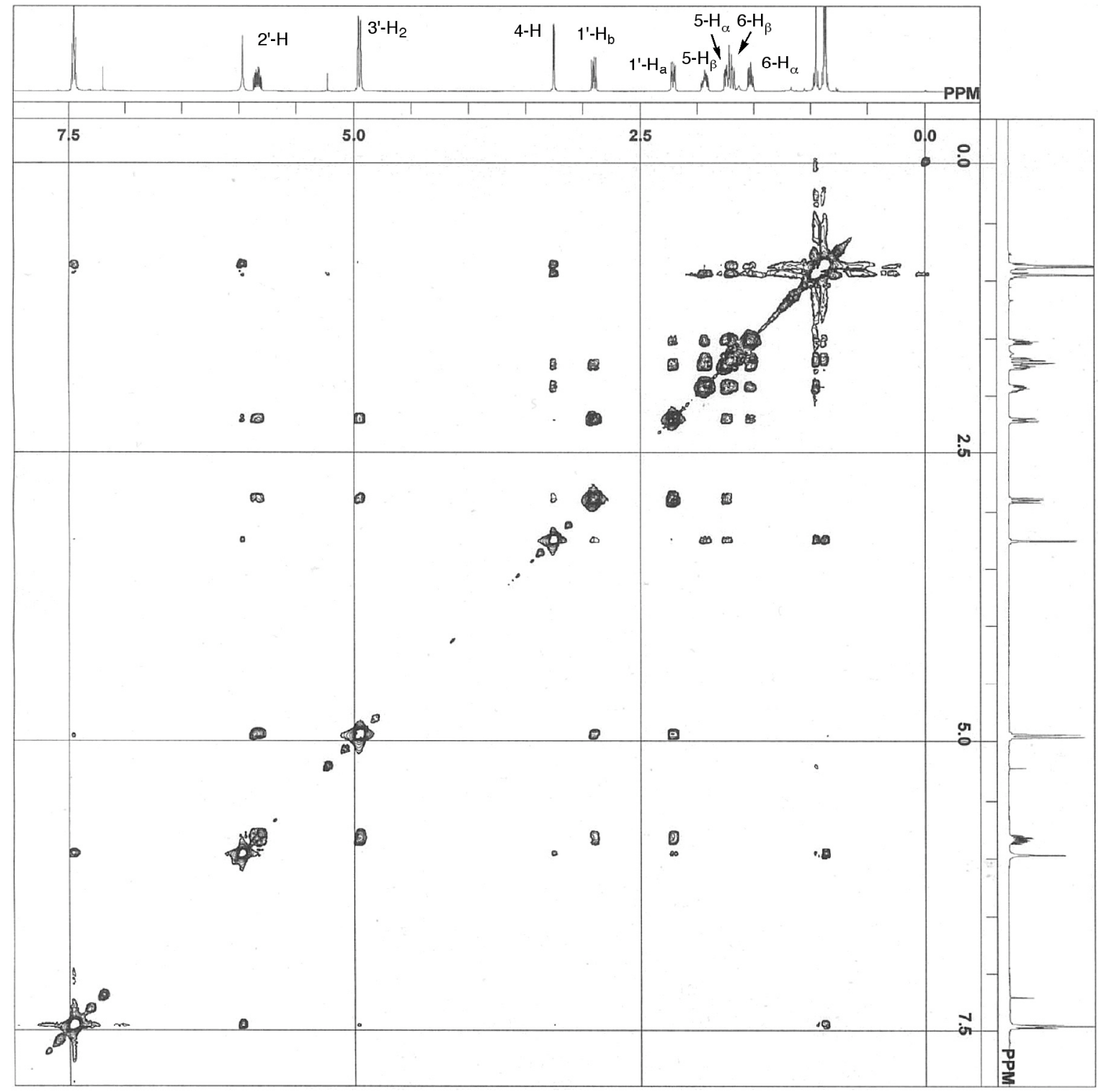

Chart S-1. NOESY spectrum of $p$-bromobenzamide of $\mathbf{1 a}$ 


\section{p-Bromobenzamide of $1 \mathrm{a}$}

${ }^{1} \mathrm{H}$ NMR $\left(600 \mathrm{MHz}, \mathrm{CDCl}_{3}\right) \delta$ 7.52-7.47 (m, 4H; Ph), 6.01 (brs, $\left.1 \mathrm{H} ; \mathrm{NH}\right), 5.88$ (dddd, $J=$ 17.8, 9.6, 8.9, 5.5 Hz, 1H; 2'-H), 5.02-4.97 (m, 2H; 3'-H), 3.30 (d, J=4.1 Hz, 1H; 4-H), 2.95 (dd, $\left.J=14.5,8.9 \mathrm{~Hz}, 1 \mathrm{H} ; 1^{\prime}-\mathrm{H}_{\mathrm{b}}\right), 2.26\left(\mathrm{dd}, J=14.5,5.5 \mathrm{~Hz}, 1 \mathrm{H} ; 1^{\prime}-\mathrm{H}_{\mathrm{a}}\right), 1.98$ (dddd, $J=13.7$, $\left.11.4,5.5,4.1 \mathrm{~Hz}, 1 \mathrm{H} ; 5-\mathrm{H}_{\beta}\right), 1.82-1.71\left(\mathrm{~m}, 2 \mathrm{H} ; 5-\mathrm{H}_{\alpha}, 6-\mathrm{H}_{\beta}\right), 1.58(\mathrm{ddd}, J=14.4,8.9,5.5 \mathrm{~Hz}$, $\left.1 \mathrm{H} ; 6-\mathrm{H}_{\alpha}\right), 1.00\left(\mathrm{~s}, 3 \mathrm{H} ; \mathrm{CH}_{3}\right), 0.93\left(\mathrm{~s}, 3 \mathrm{H} ; \mathrm{CH}_{3}\right), 0.91\left(\mathrm{~s}, 3 \mathrm{H} ; \mathrm{CH}_{3}\right) ;{ }^{13} \mathrm{C}$ NMR $(150 \mathrm{MHz}$, $\left.\mathrm{CDCl}_{3}\right) \delta 217.7,165.7,134.1,133.1,131.8,128.3,126.0,118.7,64.3,58.6,49.1,46.6,38.9$, 29.5, 22.4, 22.0, 20.7, 9.8; ESI-HRMS calcd for $\mathrm{C}_{20} \mathrm{H}_{25} \mathrm{BrNO}_{2}\left(\mathrm{M}+\mathrm{H}^{+}\right)$390.1063, found 390.1057 .

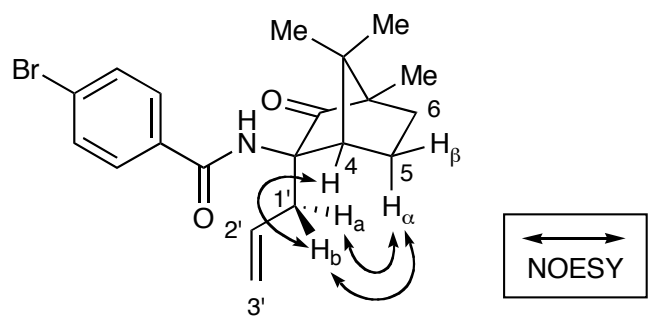

Figure S-1. The NOESY correlation of $p$-bromobenzamide of $\mathbf{1 a}$

\section{Transfer aminoallylation of glyoxylic acid (2a)}

To a solution of chiral amine 1a $(98.6 \mathrm{mg}, 0.48 \mathrm{mmol})$ in ethanol $(0.6 \mathrm{~mL})$, a solution of glyoxylic acid monohydrate (2a) $(42.8 \mathrm{mg}, 0.46 \mathrm{mmol})$ in ethanol $(0.6 \mathrm{~mL})$ was added dropwise at $-40{ }^{\circ} \mathrm{C}$. The mixture was stirred for 4 days at that temperature and quenched with $1 \mathrm{~N}$ hydrochloric acid $(5 \mathrm{~mL})$. After being stirred for $1 \mathrm{~h}$ at $\mathrm{rt}$, the mixture was washed with dichloromethane $(2 x)$ and diethyl ether $(1 \times)$. The aqueous layer was concentrated to ca. $2 \mathrm{~mL}$ and charged on a cationic ion exchange resin column (DOWEX 50W-X2, 50-100 mesh, $\mathrm{H}^{+}$form; ca. $\left.7 \mathrm{~g} / \mathrm{wet}\right)$ with purified water. After being washed with purified water $(100 \mathrm{~mL})$, the product was eluted with $1 \mathrm{~N}$ aqueous ammonia $(100 \mathrm{~mL})$. The eluant was concentrated to ca. $2 \mathrm{~mL}$ and charged on an anionic ion exchange resin column (Amberlite IRA 400, $\mathrm{OH}^{-}$ form; ca. $7 \mathrm{~g} / \mathrm{wet})$ with purified water. After being washed with purified water $(100 \mathrm{~mL})$, the product was eluted with $1 \mathrm{~N}$ hydrochloric acid $(100 \mathrm{~mL})$. The eluant was concentrated to dryness to give $(S)$-allylglycine hydrochloride (3a) (44.1 mg, 63\% yield, 93\% ee).<smiles>C=CC[C@H]([NH3+])C(=O)O[Na]</smiles>

(S)-2-Allyglycine hydrochloride (3a) 
NMR and MS data were identical to the previous report. ${ }^{2}$ The absolute configuration was assigned on comparison of the retention times of HPLC analysis with an authentic sample (obtained from Tokyo Kasei Kogyo Co., Ltd.); HPLC (CROWNPAK CR(+), 0.4 cmø $\times 25$ cmL, $0.1 \mathrm{M} \mathrm{HClO}_{4}$ aqueous solution, flow rate $0.5 \mathrm{~mL} / \mathrm{min}$, UV detection at $\left.200 \mathrm{~nm}\right) t_{R}=3.9$ $\min (R), t_{R}=5.5 \min (S)$.

\section{General procedures for transfer aminoallylation using 1a (Tables 1 and 2)}

To a solution of chiral amine $1 \mathbf{a}(103.6 \mathrm{mg}, 0.5 \mathrm{mmol})$ and an aldehyde $2(0.5 \mathrm{mmol})$ in 1,2dichloroethane $(1 \mathrm{~mL})$ was added camphorsulfonic acid $(11.6 \mathrm{mg}, 10 \mathrm{~mol} \%)$ at $0{ }^{\circ} \mathrm{C}$ or $\mathrm{rt}$. The mixture was stirred at the indicated temperature for the indicated time. Then, a solution of $\mathrm{HONH}_{2} \bullet \mathrm{AcOH}$ in methanol $\left[0.5 \mathrm{M}, 2 \mathrm{~mL}\right.$, prepared from $\mathrm{HONH}_{2} \bullet \mathrm{HCl}, \mathrm{NaOH}$ (solid, 1 equiv), and $\mathrm{AcOH}$ (1 equiv) in methanol] was added to the solution. After being stirred at $50{ }^{\circ} \mathrm{C}$ for $3 \mathrm{~h}$, the mixture was cooled to rt, acidified with $1 \mathrm{~N}$ aqueous $\mathrm{HCl}$ (pH ca. 1). The mixture was washed with dichloromethane (3x), basified with $6 \mathrm{~N}$ aqueous $\mathrm{NaOH}$ (pH ca. 10), and extracted with dichloromethane $(3 \times)$. The later dichloromethane layers were dried over anhydrous $\mathrm{Na}_{2} \mathrm{CO}_{3}$, filtered, and evaporated to give almost pure amine 3 . The crude product was purified by preparative TLC (developed with hexane/ethyl acetate $=1 / 1$ or ethyl acetate, and then eluted with hexane/isopropylamine $=10 / 1$ or $7 / 1$ ) to give amine $\mathbf{3}$.<smiles>C=CC[C@H](N)c1ccccc1</smiles>

\section{(S)-1-Phenylbut-3-en-1-amine (3b)}

Obtained from benzaldehyde (2b). NMR and MS data were identical to the previous report. ${ }^{2}$ The absolute configuration was assigned according to the literature ${ }^{3}[\alpha]^{25}-50.0$ (c 1.13, $\mathrm{CHCl}_{3}, 96 \%$ ee $(S)$ ); HPLC (CHIRALCEL OD-H, $0.46 \mathrm{~cm} \varnothing \times 25 \mathrm{cmL}$, hexane/2propanol/diethylamine $=95 / 5 / 0.05$, flow rate $1.0 \mathrm{~mL} / \mathrm{min}$, UV detection at $254 \mathrm{~nm}) t_{R}=7.3$ $\min (R), t_{R}=9.9 \min (S)$.<smiles>C=CC[C@H](N)c1ccc([N+](=O)[O-])cc1</smiles>

(S)-1-(4-Nitrophenyl)but-3-en-1-amine (3c) 
Obtained from $p$-nitrobenzaldehyde (2c). NMR and MS data were identical to the previous report. $^{2}$ The absolute configuration was assigned according to the literature ${ }^{4}:[\alpha]_{\mathrm{D}}^{22}-40.0(\mathrm{c}$ 1.52, $\mathrm{CHCl}_{3}, 97 \%$ ee $(S)$ ); HPLC (CHIRALCEL OD-H, $0.46 \mathrm{~cm} \varnothing \times 25 \mathrm{cmL}$, hexane/2propanol $/$ diethylamine $=95 / 5 / 0.05$, flow rate $1.0 \mathrm{~mL} / \mathrm{min}$, UV detection at $254 \mathrm{~nm}) t_{R}=15.5$ $\min (R), t_{R}=16.8 \min (S)$.<smiles>C=CC[C@H](N)c1ccc(Br)cc1</smiles>

\section{(S)-1-(4-Bromophenyl)but-3-en-1-amine (3d)}

Obtained from $p$-bromobenzaldehyde (2d). NMR and MS data were identical to the previous report. ${ }^{2}$ The absolute configuration was tentatively assigned on the basis of analogy: $[\alpha]^{23}{ }_{D}^{-}$ 40.1 (c 1.16, $\mathrm{CHCl}_{3}, 98 \%$ ee $(S)$ ); HPLC (CHIRALCEL OD-H, $0.46 \mathrm{~cm} \varnothing \times 25 \mathrm{cmL}$, hexane $/ 2$-propanol/diethylamine $=95 / 5 / 0.05$, flow rate $0.5 \mathrm{~mL} / \mathrm{min}$, UV detection at $254 \mathrm{~nm}$ ) $t_{R}=15.9 \min (R), t_{R}=17.1 \min (S)$.<smiles>C=CC[C@H](N)c1ccc(OC)cc1</smiles>

\section{(S)-1-(4-Methoxyphenyl)but-3-en-1-amine (3e)}

Obtained from $p$-anisaldehyde (2e). NMR and MS data were identical to the previous report. ${ }^{2}$ The absolute configuration was assigned according to the literature ${ }^{3}:[\alpha]^{23}{ }_{\mathrm{D}}-42.3$ (c 0.81 , $\mathrm{CHCl}_{3}, 97 \%$ ee $(S)$ ); HPLC (CHIRALCEL OD-H, $0.46 \mathrm{~cm} \varnothing \times 25 \mathrm{cmL}$, hexane/2propanol $/$ diethylamine $=95 / 5 / 0.05$, flow rate $1.0 \mathrm{~mL} / \mathrm{min}$, UV detection at $254 \mathrm{~nm}) t_{R}=9.8$ $\min (R), t_{R}=12.2 \min (S)$.<smiles>C=CCC(N)c1cccs1</smiles>

\section{(S)-1-(Thiophen-2-yl)but-3-en-1-amine (3f)}

Obtained from 2-thiophencarboxaldehyde (2f). NMR and MS data were identical to the previous report. ${ }^{2}$ The absolute configuration was assigned according to the literature ${ }^{3}:[\alpha]^{24}$ -23.8 (c 0.72, $\mathrm{CHCl}_{3}, 95 \%$ ee $(S)$ ); HPLC (CHIRALCEL OD-H, $0.46 \mathrm{~cm} \varnothing \times 25 \mathrm{cmL}$, 
hexane $/ 2$-propanol/diethylamine $=95 / 5 / 0.05$, flow rate $1.0 \mathrm{~mL} / \mathrm{min}$, UV detection at $254 \mathrm{~nm}$ ) $t_{R}=7.7 \min (R), t_{R}=9.4 \min (S)$.<smiles>C=CC[C@H](N)c1ccco1</smiles>

\section{(S)-1-(Furan-2-yl)but-3-en-1-amine (3g)}

Obtained from 2-furancarboxaldehyde (2g). The absolute configuration was assigned according to the literature ${ }^{3}:[\alpha]^{23}{ }_{\mathrm{D}}-37.1\left(\mathrm{c} 0.85, \mathrm{CHCl}_{3}, 94 \%\right.$ ee $\left.(S)\right) ;{ }^{1} \mathrm{H}$ NMR $(400 \mathrm{MHz}$, $\left.\mathrm{CDCl}_{3}\right) \delta 7.32(\mathrm{dd}, J=1.8,0.9 \mathrm{~Hz}, 1 \mathrm{H}), 6.28(\mathrm{dd}, J=3.2,1.8 \mathrm{~Hz}, 1 \mathrm{H}), 6.11(\mathrm{dd}, J=3.2,0.9$ $\mathrm{Hz}, 1 \mathrm{H}), 5.74$ (dddd, $J=16.9,10.1,7.4,6.8 \mathrm{~Hz}, 1 \mathrm{H}), 5.16-5.04(\mathrm{~m}, 2 \mathrm{H}), 3.98$ (dd, $J=7.8,5.5$ $\mathrm{Hz}, 1 \mathrm{H}), 2.60-2.50(\mathrm{~m}, 1 \mathrm{H}), 2.44-2.34(\mathrm{~m}, 1 \mathrm{H}), 1.53$ (brs, $2 \mathrm{H}) ;{ }^{13} \mathrm{C}$ NMR (100 $\mathrm{MHz}, \mathrm{CDCl}_{3}$ )

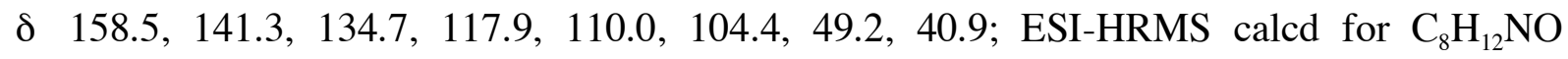
$\left(\mathrm{M}+\mathrm{H}^{+}\right)$138.0913, found 138.0918; HPLC (CROWNPAK CR(+), $0.4 \mathrm{~cm} \varnothing \times 25 \mathrm{cmL}, 0.1 \mathrm{M}$ $\mathrm{HClO}_{4}$ aqueous solution, flow rate $0.5 \mathrm{~mL} / \mathrm{min}$, UV detection at $200 \mathrm{~nm} ; t_{R}=25.9 \mathrm{~min}(S), t_{R}$ $=30.1 \min (R)$.<smiles>C=CC[C@H](N)c1cccnc1</smiles>

\section{(S)-1-(Pyridin-3-yl)but-3-en-1-amine (3h)}

Obtained from 3-pyridinecarboxaldehyde (2h). The absolute configuration was assigned according to the literature ${ }^{5}:[\alpha]^{26}{ }_{\mathrm{D}}-59.6$ (c $0.66, \mathrm{CHCl}_{3}, 98 \%$ ee $\left.(S)\right) ;{ }^{1} \mathrm{H}$ NMR $(400 \mathrm{MHz}$, $\left.\mathrm{CDCl}_{3}\right) \delta 8.56(\mathrm{~d}, J=1.8 \mathrm{~Hz}, 1 \mathrm{H}), 8.48(\mathrm{dd}, J=5.0,1.8 \mathrm{~Hz}, 1 \mathrm{H}), 7.69(\mathrm{ddd}, J=7.8,1.8,1.8$ $\mathrm{Hz}, 1 \mathrm{H}), 7.24$ (dd, $J=7.8,5.0 \mathrm{~Hz}, 1 \mathrm{H}), 5.71$ (dddd, $J=17.0,10.5,7.8,6.4 \mathrm{~Hz}, 1 \mathrm{H}), 5.17-5.06$ (m, 2H), 4.04 (dd, $J=7.8,5.5 \mathrm{~Hz}, 1 \mathrm{H}), 2.50-2.31$ (m, 2H), 1.74 (brs, $2 \mathrm{H}) ;{ }^{13} \mathrm{C}$ NMR (100 $\left.\mathrm{MHz}_{\mathrm{CDCl}}\right) \delta 148.5,140.6,134.5,133.9,123.4,118.4$, 53.0, 43.9; ESI-HRMS calcd for $\mathrm{C}_{9} \mathrm{H}_{13} \mathrm{~N}_{2}\left(\mathrm{M}+\mathrm{H}^{+}\right)$149.1073, found 149.1071; HPLC (CROWNPAK CR(+), $0.4 \mathrm{~cm} \varnothing \times 25$ cmL, $0.1 \mathrm{M} \mathrm{HClO}_{4}$ aqueous solution, flow rate $0.2 \mathrm{~mL} / \mathrm{min}$, $\mathrm{UV}$ detection at $257 \mathrm{~nm} ; t_{R}=12.0$ $\min (S), t_{R}=14.3 \min (R)$.<smiles>C=CC[C@H](N)CCc1ccccc1</smiles> 


\section{(R)-1-Phenylhex-5-en-3-amine (3i)}

Obtained from 3-phenylpropionaldehyde (2i). NMR and MS data were identical to the previous report. ${ }^{2,6}$ The absolute configuration was assigned according to the literature ${ }^{6}:[\alpha]^{25}$ +12.1 (c 0.81, $\mathrm{CHCl}_{3}, 96 \%$ ee $(R)$ ); HPLC (CHIRALCEL OD-H, $0.46 \mathrm{~cm} \varnothing \times 25 \mathrm{cmL}$, hexane $/ 2$-propanol $/$ diethylamine $=90 / 10 / 0.05$, flow rate $1.0 \mathrm{~mL} / \mathrm{min}$, UV detection at 254 $\mathrm{nm}) t_{R}=7.4 \min (S), t_{R}=10.0 \min (R)$.<smiles>C=CCC(N)Cc1ccccc1</smiles>

\section{(S)-1-Phenylpent-4-en-2-amine (3j)}

Obtained from phenylacetaldehyde $(\mathbf{2} \mathbf{j})$. The absolute configuration was tentatively assigned on the basis of analogy: $[\alpha]^{21}{ }_{\mathrm{D}}+28.3$ (c 1.59, $\mathrm{CHCl}_{3}, 93 \%$ ee $(S)$ ); ${ }^{1} \mathrm{H}$ NMR $(400 \mathrm{MHz}$, $\left.\mathrm{CDCl}_{3}\right) \delta$ 7.35-7.18 (m, 5H), 5.83 (dddd, $\left.J=17.0,10.3,7.8,6.4 \mathrm{~Hz}, 1 \mathrm{H}\right), 5.19-5.08(\mathrm{~m}, 2 \mathrm{H})$, 3.09 (dddd, $J=8.2,7.8,5.0,5.0 \mathrm{~Hz}, 1 \mathrm{H}), 2.80(\mathrm{dd}, J=13.3,5.0 \mathrm{~Hz}, 1 \mathrm{H}), 2.55(\mathrm{dd}, J=13.3$, $8.2 \mathrm{~Hz}, 1 \mathrm{H}), 2.30$ (ddd, $J=13.7,6.4,5.0 \mathrm{~Hz}, 1 \mathrm{H}), 2.09$ (ddd, $J=13.7,7.8,7.8 \mathrm{~Hz}, 1 \mathrm{H}), 1.66$ (brs, $2 \mathrm{H}) ;{ }^{13} \mathrm{C} \mathrm{NMR}\left(100 \mathrm{MHz}, \mathrm{CDCl}_{3}\right) \delta 139.4,135.6,129.3,128.5,126.3,117.6,52.1,44.1$, 41.9; ESI-HRMS calcd for $\mathrm{C}_{11} \mathrm{H}_{16} \mathrm{~N} \quad\left(\mathrm{M}+\mathrm{H}^{+}\right)$162.1277, found 162.1278; HPLC (CHIRALCEL OD-H, $0.46 \mathrm{~cm} \varnothing \times 25 \mathrm{cmL}$, hexane/2-propanol/diethylamine = 99.8/0.2/0.05, flow rate $1.0 \mathrm{~mL} / \mathrm{min}$, UV detection at $254 \mathrm{~nm}) t_{R}=13.5 \mathrm{~min}(S), t_{R}=14.9 \mathrm{~min}(R)$.<smiles>C=CC[C@H](N)CCCCCCCCC</smiles>

\section{(R)-Undec-1-en-4-amine (3k)}

Obtained from octanal (2k). The absolute configuration was tentatively assigned on the basis of analogy: $[\alpha]_{\mathrm{D}}^{27}+5.9$ (c $1.17, \mathrm{CHCl}_{3}, 93 \%$ ee $\left.(R)\right) ;{ }^{1} \mathrm{H} \mathrm{NMR}\left(400 \mathrm{MHz}, \mathrm{CDCl}_{3}\right) \delta 5.77$ (dddd, $J=16.7,10.5,8.2,6.4 \mathrm{~Hz}, 1 \mathrm{H}), 5.10-5.02$ (m, 2H), 2.78-2.69 (m, 1H), 2.25-2.15 (m, $1 \mathrm{H}), 1.99-1.89(\mathrm{~m}, 1 \mathrm{H}), 1.44-1.14(\mathrm{~m}, 14 \mathrm{H}), 0.85(\mathrm{t}, J=6.9 \mathrm{~Hz}, 3 \mathrm{H}) ;{ }^{13} \mathrm{C} \mathrm{NMR}(100 \mathrm{MHz}$, $\left.\mathrm{CDCl}_{3}\right) \delta 136.0,117.1,50.6,42.6,37.7,31.8,29.7,29.3,26.2,22.6,14.1$; ESI-HRMS calcd for $\mathrm{C}_{11} \mathrm{H}_{24} \mathrm{~N}\left(\mathrm{M}+\mathrm{H}^{+}\right)$170.1903, found 170.1902.

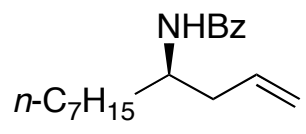

\section{Benzamide of $3 \mathbf{k}$}


For determination of the enantiomeric excess, 3k was converted to the corresponding benzamide under the usual procedure [benzoyl chloride (1.2 equiv) and triethylamine (1.5 equiv) in dichloromethane at $\mathrm{rt}$ ]: ${ }^{1} \mathrm{H} \mathrm{NMR}\left(400 \mathrm{MHz}, \mathrm{CDCl}_{3}\right) \delta 7.72$ (apparent $\mathrm{d}, J=8.0 \mathrm{~Hz}$, 2H), 7.50-7.35 (m, 3H), $5.94(\mathrm{~d}, J=8.7 \mathrm{~Hz}, 1 \mathrm{H}), 5.81$ (dddd, $J=17.4,10.3,6.9,6.0 \mathrm{~Hz}, 1 \mathrm{H})$, 5.13-5.05 (m, 2H), 4.25-4.13 (m, 1H), 2.36 (ddd, $J=14.0,6.0,6.0 \mathrm{~Hz}, 1 \mathrm{H}), 2.28$ (ddd, $J=$ $14.0,6.9,6.9 \mathrm{~Hz}, 1 \mathrm{H}), 1.63-1.42(\mathrm{~m}, 2 \mathrm{H}), 1.42-1.17(\mathrm{~m}, 10 \mathrm{H}), 0.84(\mathrm{t}, J=6.6 \mathrm{~Hz}, 3 \mathrm{H}) ;{ }^{13} \mathrm{C}$ NMR $\left(100 \mathrm{MHz}, \mathrm{CDCl}_{3}\right) \delta 167.0,135.0,134.4,131.2,128.5,126.8,117.9,49.0,39.2,34.5$, 31.7, 29.5, 29.2, 26.0, 22.6, 14.0; ESI-HRMS calcd for $\mathrm{C}_{18} \mathrm{H}_{28} \mathrm{NO}\left(\mathrm{M}+\mathrm{H}^{+}\right)$274.2165, found 274.2167; HPLC (CHIRALCEL OD-H, $0.46 \mathrm{~cm} \varnothing \times 25 \mathrm{cmL}$, hexane/2-propanol/diethylamine $=95 / 5 / 0.05$, flow rate $1.0 \mathrm{~mL} / \mathrm{min}$, UV detection at $254 \mathrm{~nm}) t_{R}=9.2 \min (R), t_{R}=12.3 \mathrm{~min}(S)$.<smiles>C=CC[C@H](N)C[C@H](C)CCC=C(C)C</smiles>

\section{(4S,6S)-6,10-Dimethylundeca-1,9-dien-4-amine (3l)}

Obtained from $(S)$-citronellal (2l) [95\% ee $(S)]$. Due to the high hydrophobicity of 31, it was difficult to extract $\mathbf{3 l}$ completely into the acidic aqueous layer. Therefore, $\mathbf{3 l}$ was extracted with dichloromethane directly from the reaction mixture (basified with $6 \mathrm{~N}$ aqueous $\mathrm{NaOH}$ ), and then purified by PTLC. The relative configuration was tentatively assigned on the basis of analogy, and the diastereomeric excess was estimated by ${ }^{13} \mathrm{C}$ NMR analysis (93\% de): $[\alpha]_{\mathrm{D}}^{23}+15.9$ (c 0.99, $\left.\mathrm{CHCl}_{3}, 93 \% \mathrm{de}\right) ;{ }^{1} \mathrm{H} \mathrm{NMR}\left(600 \mathrm{MHz}, \mathrm{CDCl}_{3}\right) \delta 5.79$ (dddd, $J=16.5$, $10.3,8.0,6.5 \mathrm{~Hz}, 1 \mathrm{H}), 5.12-5.06(\mathrm{~m}, 3 \mathrm{H}), 2.88$ (ddt, $J=8.9,8.0,4.6 \mathrm{~Hz}, 1 \mathrm{H}), 2.23-2.16$ (m, $1 \mathrm{H}), 2.05-1.92(\mathrm{~m}, 3 \mathrm{H}), 1.66$ (s, 3H), 1.60 (brs, 4H), 1.46-1.34 (m, 2H), 1.34-1.26 (m, 2H), $1.22-1.13(\mathrm{~m}, 2 \mathrm{H}), 0.89$ (d, $J=6.2 \mathrm{~Hz}, 3 \mathrm{H}) ;{ }^{13} \mathrm{C} \mathrm{NMR}\left(150 \mathrm{MHz}, \mathrm{CDCl}_{3}\right) \delta 135.91,131.13$, 124.73, 117.17, 47.90, 45.14, 43.45, 37.89, 29.15, 25.66, 25.44, 19.09, 17.61; ESI-HRMS calcd for $\mathrm{C}_{13} \mathrm{H}_{26} \mathrm{~N}\left(\mathrm{M}+\mathrm{H}^{+}\right)$196.2060, found 196.2060.

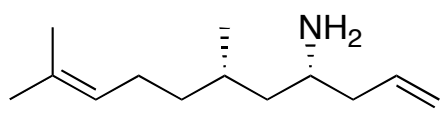

\section{(4R,6S)-6,10-Dimethylundeca-1,9-dien-4-amine (3I)}

Obtained from $(S)$-citronellal (2l) [95\% ee $(S)$ ] and the enantiomer of 1a [prepared from $(1 S)$ camphorquinone]. The product was isolated as described above. The relative configuration was tentatively assigned on the basis of analogy, and the diastereomeric excess was estimated by ${ }^{13} \mathrm{C}$ NMR analysis $\left(92 \%\right.$ de): $[\alpha]^{23}{ }_{\mathrm{D}}-3.9$ (c $0.99, \mathrm{CHCl}_{3}, 91 \%$ de); ${ }^{1} \mathrm{H}$ NMR $(600 \mathrm{MHz}$, $\mathrm{CDCl}_{3}$ ) $\delta 5.79$ (dddd, $J=16.5,10.6,8.3,6.5 \mathrm{~Hz}, 1 \mathrm{H}$ ), 5.14-5.06 (m, 3H), 2.89 (apparent ddt, $J$ 
= 7.6, 6.8, 4.1 Hz, 1H), 2.28-2.20 (m, 1H), 2.06-1.89 (m, 3H), $1.68(\mathrm{~s}, 3 \mathrm{H}), 1.60(\mathrm{~s}, 3 \mathrm{H}), 1.59$ $1.52(\mathrm{~m}, 3 \mathrm{H}), 1.40-1.08(\mathrm{~m}, 4 \mathrm{H}), 0.91(\mathrm{~d}, J=6.8 \mathrm{~Hz}, 3 \mathrm{H}) ;{ }^{13} \mathrm{C} \mathrm{NMR}\left(150 \mathrm{MHz}, \mathrm{CDCl}_{3}\right) \delta$ 135.79, 131.17, 124.71, 117.28, 48.03, 45.30, 42.45, 36.66, 29.24, 25.65, 25.26, 20.15, 17.60; ESI-HRMS calcd for $\mathrm{C}_{13} \mathrm{H}_{26} \mathrm{~N}\left(\mathrm{M}+\mathrm{H}^{+}\right)$196.2060, found 196.2053.

\section{Determination of the enantiomeric excess of $(S)$-citronellal (2l)}

The enantiomeric excess of $(S)$-citronellal (purchased from Aldrich Chemical Company) was determined by HPLC analysis after conversion to the corresponding $N$ '-benzoylhydrazone [treatment with $\mathrm{N}$-benzoylhydrazine (1 equiv) in THF at $\mathrm{rt}$ for $5 \mathrm{~h}$ ]. HPLC (CHIRALCEL OD, $0.46 \mathrm{~cm} \varnothing \times 25 \mathrm{cmL}$, hexane $/ 2$-propanol $=19 / 1$, flow rate $1.0 \mathrm{~mL} / \mathrm{min}$, UV detection at $254 \mathrm{~nm}) t_{R}=30.3 \min (S), t_{R}=35.7 \min (R)$.<smiles>C=CC[C@H](N)C1CCCCC1</smiles>

\section{(S)-1-Cyclohexylbut-3-en-1-amine (3m)}

Obtained from cyclohexanecarboxaldehyde (2m). NMR and MS data were identical to the previous report. $^{2}$ The absolute configuration was assigned according to the literature ${ }^{4}[\alpha]^{26}$ +4.5 (c $1.04, \mathrm{CHCl}_{3}, 95 \%$ ee $\left.(S)\right)$.<smiles>C=CCC(N)C1CCCCC1</smiles>

\section{Benzamide of $3 \mathrm{~m}$}

For determination of the enantiomeric excess, 3m was converted to the corresponding benzamide under the usual procedure (see the procedure for $3 \mathbf{k}):{ }^{1} \mathrm{H}$ NMR (400 $\mathrm{MHz}, \mathrm{CDCl}_{3}$ ) $\delta 7.72(\mathrm{dd}, J=8.2,1.4 \mathrm{~Hz}, 2 \mathrm{H}), 7.50-7.37(\mathrm{~m}, 3 \mathrm{H}), 5.91(\mathrm{brd}, J=9.2 \mathrm{~Hz}, 1 \mathrm{H}), 5.80$ (dddd, $J$ $=17.2,10.0,7.3,6.9 \mathrm{~Hz}, 1 \mathrm{H}), 5.14-4.99(\mathrm{~m}, 2 \mathrm{H}), 4.15-4.02(\mathrm{~m}, 1 \mathrm{H}), 2.39$ (ddd, $J=14.4,6.9$, $5.5 \mathrm{~Hz}, 1 \mathrm{H}), 2.26(\mathrm{ddd}, J=14.4,7.3,7.3 \mathrm{~Hz}, 1 \mathrm{H}), 1.83-1.69(\mathrm{~m}, 4 \mathrm{H}), 1.64(\mathrm{brd}, J=11.0 \mathrm{~Hz}$, $1 \mathrm{H}), 1.55-1.42(\mathrm{~m}, 1 \mathrm{H}), 1.29-0.95(\mathrm{~m}, 5 \mathrm{H}) ;{ }^{13} \mathrm{C} \mathrm{NMR}\left(100 \mathrm{MHz}, \mathrm{CDCl}_{3}\right) \delta 167.1,135.1$, 134.8, 131.2, 128.5, 126.8, 117.6, 53.2, 41.3, 36.4, 29.7, 28.8, 26.3, 26.14, 26.12; ESI-HRMS calcd for $\mathrm{C}_{17} \mathrm{H}_{24} \mathrm{NO}\left(\mathrm{M}+\mathrm{H}^{+}\right)$258.1852, found 258.1857; HPLC (CHIRALCEL OD-H, 0.46 $\mathrm{cm} \varnothing \times 25 \mathrm{cmL}$, hexane $/ 2$-propanol/diethylamine $=95 / 5 / 0.05$, flow rate $0.5 \mathrm{~mL} / \mathrm{min}, \mathrm{UV}$ detection at $254 \mathrm{~nm}) t_{R}=18.8 \min (R), t_{R}=20.3 \min (S)$. 
$\overbrace{\mathrm{NnO}}^{\mathrm{NH}_{2}}$

\section{(S)-1-(Benzyloxy)hex-5-en-3-amine (3n)}

Obtained from 3-(benzyloxy)propanal (2n). The absolute configuration was tentatively assigned on the basis of analogy: $[\alpha]_{\mathrm{D}}^{25}+26.8$ (c 1.27, $\mathrm{CHCl}_{3}, 96 \%$ ee $(S)$ ); ${ }^{1} \mathrm{H}$ NMR (400 $\left.\mathrm{MHz} \mathrm{CDCl}_{3}\right) \delta$ 7.36-7.22 (m, 5H), 5.76 (dddd, $\left.J=15.4,11.7,7.8,6.9 \mathrm{~Hz}, 1 \mathrm{H}\right), 5.10-5.02(\mathrm{~m}$, 2H), 4.49 (s, 2H), 3.63-3.52 (m, 2H), 2.97 (dddd, $J=8.7,8.0,4.7,4.6 \mathrm{~Hz}, 1 \mathrm{H}), 2.21$ (ddd, $J=$ 13.7, 6.9, $4.7 \mathrm{~Hz}, 1 \mathrm{H}), 1.99$ (ddd, $J=13.7,8.0,7.8 \mathrm{~Hz}, 1 \mathrm{H}$ ), 1.75 (dddd, $J=14.2,6.9,6.9,4.6$ $\mathrm{Hz}, 1 \mathrm{H}$ ), 1.55 (dddd, $J=14.2,8.7,6.0,6.0 \mathrm{~Hz}, 1 \mathrm{H}), 1.29$ (brs, 2H); ${ }^{13} \mathrm{C}$ NMR (100 MHz, $\left.\mathrm{CDCl}_{3}\right) \delta 138.4,135.6,128.3,127.6,127.5,117.4,73.0,68.0,48.4,42.8,37.3$; ESI-HRMS calcd for $\mathrm{C}_{13} \mathrm{H}_{20} \mathrm{NO}\left(\mathrm{M}+\mathrm{H}^{+}\right)$206.1539, found 206.1542; HPLC (CHIRALCEL OD-H, 0.46 $\mathrm{cm} \varnothing \times 25 \mathrm{cmL}$, hexane/2-propanol/diethylamine $=95 / 5 / 0.05$, flow rate $1.0 \mathrm{~mL} / \mathrm{min}, \mathrm{UV}$ detection at $254 \mathrm{~nm}) t_{R}=8.2 \min (S), t_{R}=9.2 \min (R)$.<smiles>C=CCC(N)CO[B]Cc1ccccc1</smiles>

\section{(S)-1-(Benzyloxy)pent-4-en-2-amine (3o)}

Obtained from 3-(benzyloxy)acetaldehyde (2o). The absolute configuration was tentatively assigned on the basis of analogy: $[\alpha]^{25}{ }_{\mathrm{D}}+7.1$ (c 1.14, $\mathrm{CHCl}_{3}, 92 \%$ ee $(S)$ ); ${ }^{1} \mathrm{H}$ NMR $(400$ $\mathrm{MHz}, \mathrm{CDCl}_{3}$ ) $\delta$ 7.12-6.97 (m, 5H), 5.52 (dddd, $\left.J=17.0,10.1,7.6,6.9 \mathrm{~Hz}, 1 \mathrm{H}\right), 4.88-4.77$ (m, 2H), 4.26 (s, 2H), 3.19 (dd, $J=8.7,4.1 \mathrm{~Hz}, 1 \mathrm{H}), 3.02$ (dd, $J=8.7,7.8 \mathrm{~Hz}, 1 \mathrm{H}), 2.80$ (dddd, $J$ $=7.8,7.8,5.3,4.1 \mathrm{~Hz}, 1 \mathrm{H}), 1.97(\mathrm{ddd}, J=13.8,6.9,5.3 \mathrm{~Hz}, 1 \mathrm{H}), 1.78(\mathrm{ddd}, J=13.8,7.8,7.6$ $\mathrm{Hz}, 1 \mathrm{H}), 1.15$ (s, 1H); ${ }^{13} \mathrm{C} \mathrm{NMR}\left(100 \mathrm{MHz}, \mathrm{CDCl}_{3}\right) \delta 138.3,135.2,128.3,127.6,127.5,117.4$, 75.3, 73.2, 50.3, 38.8; ESI-HRMS calcd for $\mathrm{C}_{12} \mathrm{H}_{18} \mathrm{NO}\left(\mathrm{M}+\mathrm{H}^{+}\right)$192.1383, found 192.1382; HPLC (CHIRALCEL OD-H, $0.46 \mathrm{~cm} \varnothing \times 25 \mathrm{cmL}$, hexane/2-propanol/diethylamine = $95 / 5 / 0.05$, flow rate $1.0 \mathrm{~mL} / \mathrm{min}$, UV detection at $254 \mathrm{~nm}) t_{R}=9.8 \mathrm{~min}(R), t_{R}=11.7 \mathrm{~min}(S)$.

\section{Isolation of intermediate $4 \mathrm{~b}$}

A mixture of chiral amine $1 \mathbf{a}(103.3 \mathrm{mg}, 0.5 \mathrm{mmol})$ and benzaldehyde $(\mathbf{2 b})(0.6 \mathrm{mmol})$, in 1,2-dichloroethane $(1 \mathrm{~mL})$ was stirred at $50{ }^{\circ} \mathrm{C}$ for $6 \mathrm{~h}$. After evaporation of the solvent, the residue was purified by neutral silica gel column chromatography (hexane/ethyl acetate $=10$ /1) to give imine $\mathbf{4 b}$ (130.4 $\mathrm{mg}, 89 \%$ yield). 


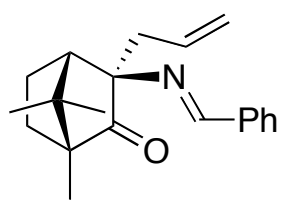

$(E, 1 R, 3 R, 4 S)$-3-Allyl-3-(benzylideneamino)-1,7,7-trimethylbicyclo[2.2.1]heptan-2-one

(4b). ${ }^{1} \mathrm{H}$ NMR (400 MHz, $\mathrm{CDCl}_{3}$ ) $\delta 8.57$ (s, 1H), 7.74-7.67 (m, 2H), 7.40-7.34 (m, 3H), 5.73 (dddd, $J=17.0,10.1,7.3,6.9 \mathrm{~Hz}, 1 \mathrm{H}), 5.06-4.95(\mathrm{~m}, 2 \mathrm{H}), 2.56(\mathrm{dd}, J=14.8,6.9 \mathrm{~Hz}, 1 \mathrm{H})$, $2.52(\mathrm{~d}, J=3.7 \mathrm{~Hz}, 1 \mathrm{H}), 2.40(\mathrm{dd}, J=14.8,7.3 \mathrm{~Hz}, 1 \mathrm{H}), 2.03-1.91(\mathrm{~m}, 1 \mathrm{H}), 1.81-1.68(\mathrm{~m}$, $2 \mathrm{H}), 1.56-1.48(\mathrm{~m}, 1 \mathrm{H}), 1.01(\mathrm{~s}, 3 \mathrm{H}), 0.91(\mathrm{~s}, 3 \mathrm{H}), 0.75$ (s, 3H); ${ }^{13} \mathrm{C} \mathrm{NMR}\left(100 \mathrm{MHz}, \mathrm{CDCl}_{3}\right)$ ठ $219.9,159.5,136.7,132.5,130.5,128.4,128.0,118.8,71.9,58.3,51.3,46.7,42.1,31.3$, 22.7, 22.2, 20.6, 9.7; ESI-HRMS calcd for $\mathrm{C}_{20} \mathrm{H}_{26} \mathrm{NO}\left(\mathrm{M}+\mathrm{H}^{+}\right)$296.2009, found 296.2006.

\section{Isolation of intermediate $5 \mathbf{b}$}

A mixture of chiral amine 1a $(189.8 \mathrm{mg}, 0.9 \mathrm{mmol})$, benzaldehyde $(\mathbf{2 b})(0.9 \mathrm{mmol})$, and camphorsulfonic acid $(22.4 \mathrm{mg}, 11 \mathrm{~mol} \%)$ in 1,2-dichloroethane $(1.8 \mathrm{~mL})$ was stirred at $50{ }^{\circ} \mathrm{C}$ for $48 \mathrm{~h}$. After evaporation of the solvent, the residue was directly purified by PTLC (hexane/ethyl acetate $=6 / 1)$ to give rearranged products $(E)-5 \mathbf{b}(182.6 \mathrm{mg}, 68 \%$ yield $)$ and (Z)-5b (27.3 mg, 10\% yield).

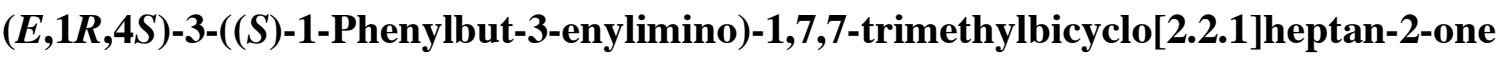

[(E)-5b] ${ }^{1} \mathrm{H}$ NMR $\left(600 \mathrm{MHz}, \mathrm{C}_{6} \mathrm{D}_{6}\right) \delta 7.32$ (apparent d, $\left.J=8.2 \mathrm{~Hz}, 2 \mathrm{H} ; \mathrm{Ph}\right), 7.05$ (apparent t, $J=7.9 \mathrm{~Hz}, 2 \mathrm{H} ; \mathrm{Ph}$ ), 6.95 (apparent t, $J=7.6 \mathrm{~Hz}, 1 \mathrm{H} ; \mathrm{Ph}$ ), 5.57 (dddd, $J=17.2,10.3$, 7.6, 6.9 $\mathrm{Hz}, 1 \mathrm{H} ; 3$ '-H), 4.88 (dddd, $J=17.2,2.0,1.4,1.4 \mathrm{~Hz}, 1 \mathrm{H} ; 4^{\prime}-\mathrm{H}_{\text {trans }}$ ), 4.81 (apparent dd, $J=10.3$, $2.0 \mathrm{~Hz}, 1 \mathrm{H} ; 4^{\prime}-\mathrm{H}_{\mathrm{cis}}$ ), 4.23 (dd, $\left.J=8.3,5.5 \mathrm{~Hz}, 1 \mathrm{H} ; 1^{\prime}-\mathrm{H}\right), 2.70-2.64$ (m, 1H; 2'- $\mathrm{H}_{\mathrm{a}}$ ), 2.54-2.48 (m, 1H; 2'- $\mathrm{H}_{\mathrm{b}}$ ), 2.41 (d, $\left.J=4.8 \mathrm{~Hz}, 1 \mathrm{H} ; 4-\mathrm{H}\right), 1.21$ (dddd, $J=13.1,11.7,4.8,4.8 \mathrm{~Hz}, 1 \mathrm{H} ; 5-$ $\mathrm{H}_{\beta}$ ), 1.01 (ddd, $\left.J=13.7,11.7,4.1 \mathrm{~Hz}, 1 \mathrm{H} ; 6-\mathrm{H}_{\beta}\right), 0.84$ (ddd, $\left.J=13.7,8.9,4.8 \mathrm{~Hz}, 1 \mathrm{H} ; 6-\mathrm{H}_{\alpha}\right)$, $0.78\left(\mathrm{~s}, 3 \mathrm{H} ; 1-\mathrm{CH}_{3}\right), 0.63\left(\mathrm{ddd}, J=13.1,8.9,4.1 \mathrm{~Hz}, 1 \mathrm{H} ; 5-\mathrm{H}_{\alpha}\right), 0.55\left(\mathrm{~s}, 3 \mathrm{H} ; 7-\mathrm{CH}_{3}\right), 0.40$ (s, $\left.3 \mathrm{H} ; 7-\mathrm{CH}_{3}\right) ;{ }^{13} \mathrm{C}$ NMR $\left(150 \mathrm{MHz}, \mathrm{C}_{6} \mathrm{D}_{6}\right) \delta 204.4,170.1,143.7,135.6,128.3,127.7,127.5$, 117.3, 68.1, 57.6, 49.0, 43.92, 43.88, 29.8, 23.5, 20.9, 17.5, 9.2; ESI-HRMS calcd for $\mathrm{C}_{20} \mathrm{H}_{26} \mathrm{NO}\left(\mathrm{M}+\mathrm{H}^{+}\right)$296.2009, found 296.2005. The configuration was determined by a NOESY experiment (Chart S-2).

\section{$(Z, 1 R, 4 S)-3-((S)-1-P h e n y l b u t-3-e n y l i m i n o)-1,7,7-t r i m e t h y l b i c y c l o[2.2 .1] h e p t a n-2-o n e$} [(Z)-5b] ${ }^{1} \mathrm{H}$ NMR $\left(600 \mathrm{MHz}, \mathrm{C}_{6} \mathrm{D}_{6}\right) \delta 7.49$ (apparent d, $\left.J=8.2 \mathrm{~Hz}, 2 \mathrm{H} ; \mathrm{Ph}\right), 7.06$ (apparent t, 
$J=7.9 \mathrm{~Hz}, 2 \mathrm{H} ; \mathrm{Ph}), 6.93($ apparent t $, J=7.6 \mathrm{~Hz}, 1 \mathrm{H} ; \mathrm{Ph}), 6.36(\mathrm{dd}, J=8.2,4.9 \mathrm{~Hz}, 1 \mathrm{H} ; 1$ '-H), 5.71 (dddd, $J=17.2,10.3,7.6,6.2 \mathrm{~Hz}, 1 \mathrm{H} ; 3^{\prime}-\mathrm{H}$ ), 4.90 (dddd, $J=17.2,2.1,1.4,1.4 \mathrm{~Hz}, 1 \mathrm{H}$; 4'- $\mathrm{H}_{\text {trans }}$ ), 4.82 (apparent dd, $J=10.3,2.1 \mathrm{~Hz}, 1 \mathrm{H} ; 4^{\prime}-\mathrm{H}_{\text {cis }}$ ), 2.70-2.42 (m, 2H; 2'-H), 2.35 (d, $J$ $=4.8 \mathrm{~Hz}, 1 \mathrm{H} ; 4-\mathrm{H}), 1.56$ (dddd, $J=13.1,11.7,5.5,4.8 \mathrm{~Hz}, 1 \mathrm{H} ; 5-\mathrm{H}_{\beta}$ ), 1.36 (ddd, $J=13.1,8.9$, $4.8 \mathrm{~Hz}, 1 \mathrm{H} ; 5-\mathrm{H}_{\alpha}$ ), 1.17 (ddd, $J=13.7,11.7,4.8 \mathrm{~Hz}, 1 \mathrm{H} ; 6-\mathrm{H}_{\mathrm{a}}$ ), 1.13 (ddd, $J=13.7,8.9,5.5$ $\left.\mathrm{Hz}, 1 \mathrm{H} ; 5-\mathrm{H}_{\mathrm{b}}\right), 0.69\left(\mathrm{~s}, 3 \mathrm{H} ; 1-\mathrm{CH}_{3}\right), 0.331\left(\mathrm{~s}, 3 \mathrm{H} ; 7-\mathrm{CH}_{3}\right), 0.325\left(\mathrm{~s}, 3 \mathrm{H} ; 7-\mathrm{CH}_{3}\right) ;{ }^{13} \mathrm{C} \mathrm{NMR}$ $\left(150 \mathrm{MHz}, \mathrm{C}_{6} \mathrm{D}_{6}\right) \delta$ 203.6, 167.4, 144.7, 135.9, 128.6, 127.7, 127.1, 117.0, 62.5, 59.9, 55.5, $44.4,43.8,29.9,25.4,20.1,17.7,9.2$. The configuration was determined by a NOESY experiment (Chart S-3).

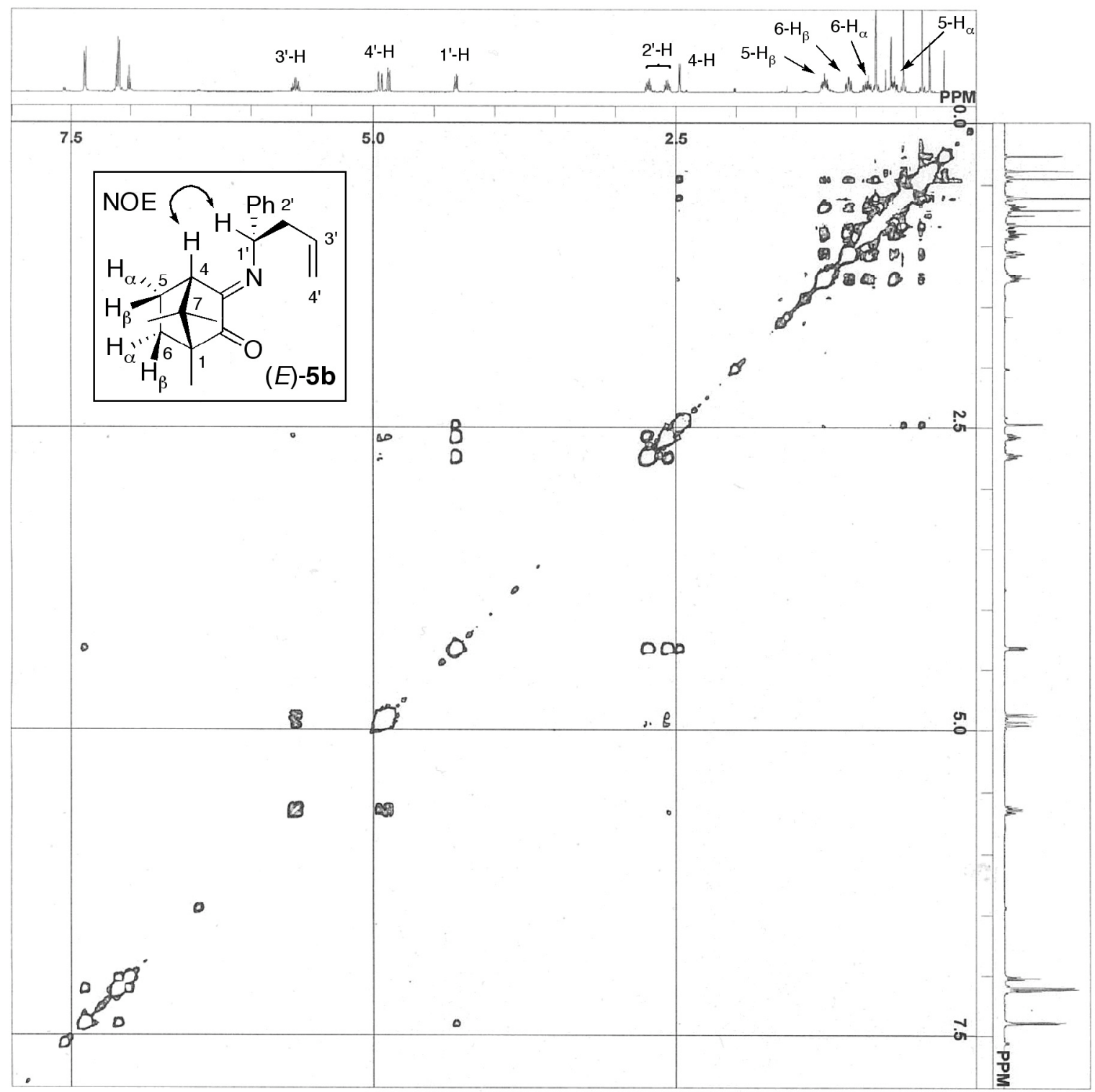

Chart S-2. NOESY spectrum of $(E)-\mathbf{5 b}(Z: E=5: 95)$ 


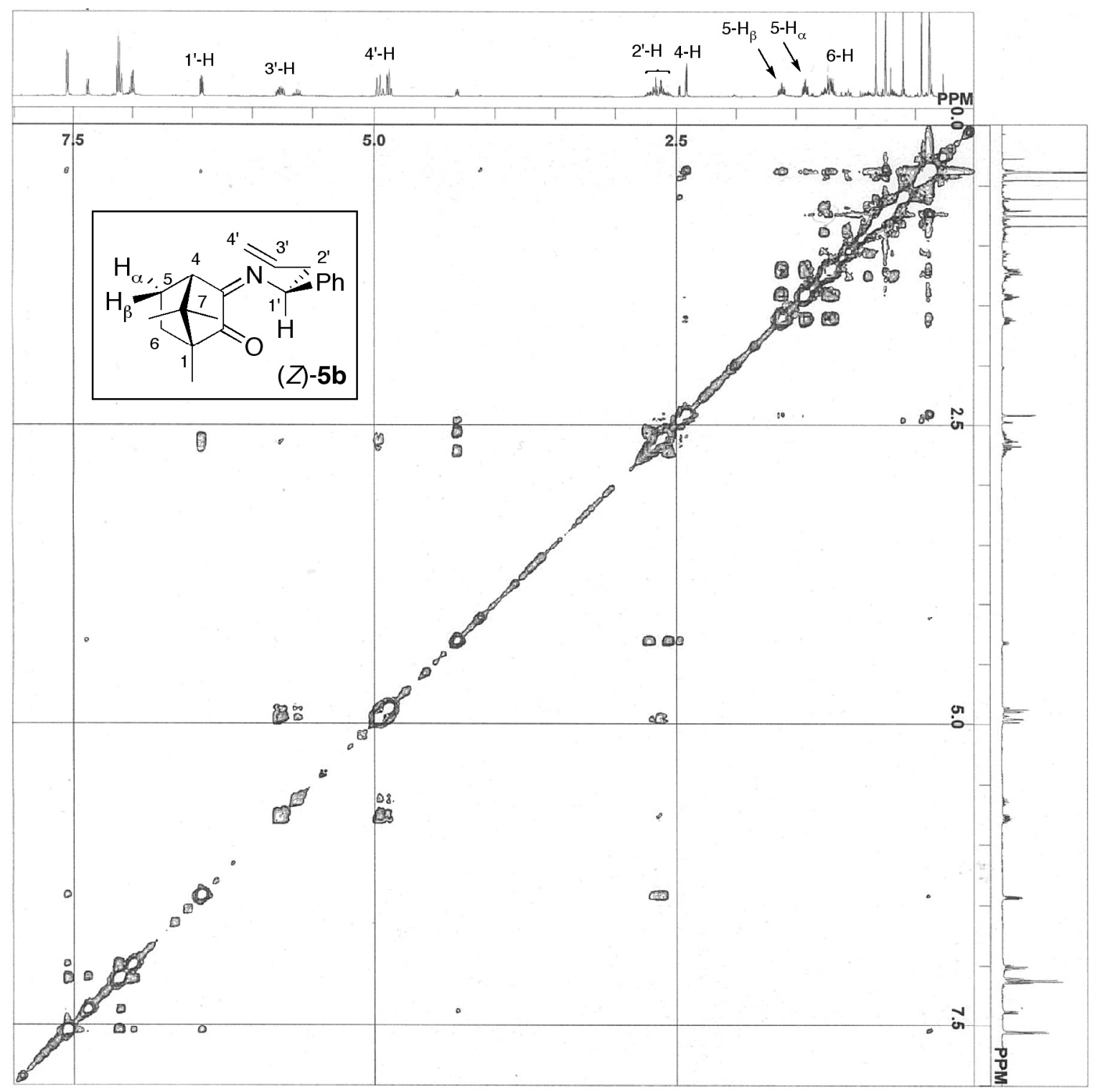

Chart S-3. NOESY spectrum of (Z)-5b $(Z: E=80: 20)$

\section{NMR analysis of reaction of $4 \mathrm{~b}$ in $\mathrm{CDCl}_{3}$}

A reaction of imine $\mathbf{4 b}(127.1 \mathrm{mg}, 0.43 \mathrm{mmol})$ and camphorsulfonic acid $(9.1 \mathrm{mg}, 9 \mathrm{~mol} \%)$ in deuterated chloroform $(0.9 \mathrm{~mL})$ at $50{ }^{\circ} \mathrm{C}$ was monitored by ${ }^{1} \mathrm{H}$ NMR spectroscopy until $48 \mathrm{~h}$ (Chart S-4). The reaction reached equilibrium within 48 h [2b:4b:(Z)-5b:(E)-5b = 2:6:18:74].
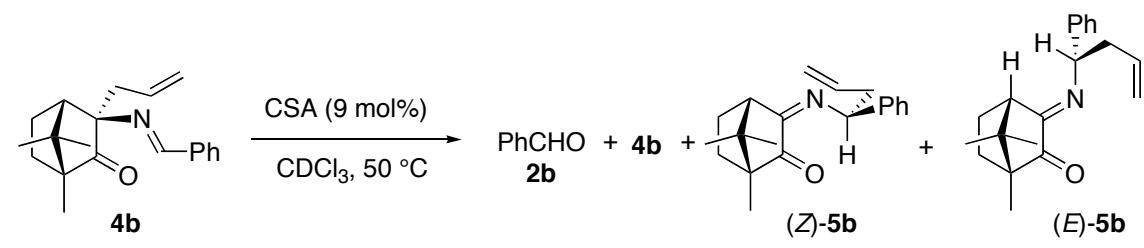

$2: 6: 18: 74$ 


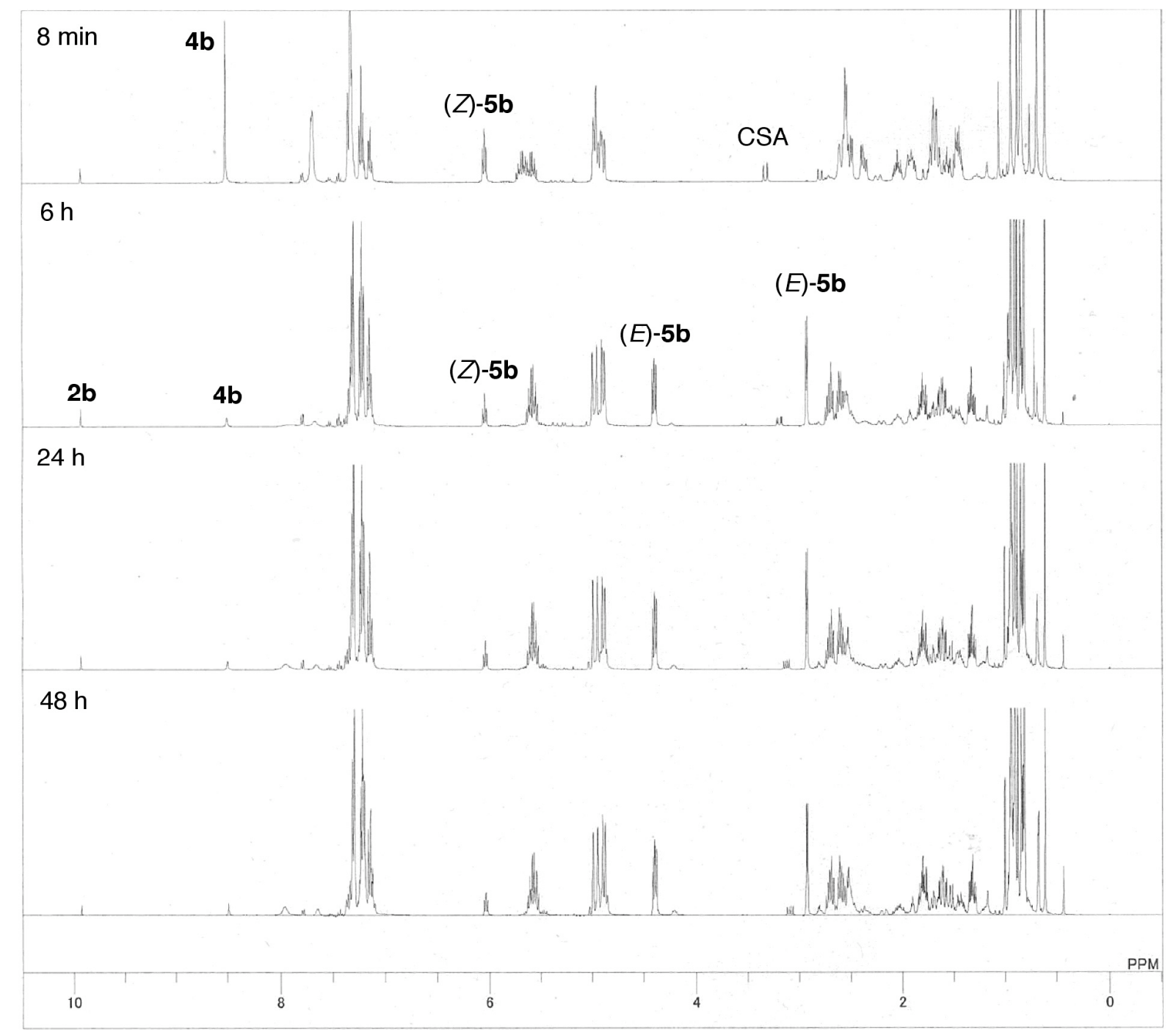

Chart S-4. NMR monitoring of reaction of $\mathbf{4 b}$

\section{NMR analysis of reaction of $5 \mathrm{~b}$ in $\mathrm{CDCl}_{3}$}

A reaction of imine $\mathbf{5 b}(104.7 \mathrm{mg}, 0.35 \mathrm{mmol})$ and camphorsulfonic acid (7.6 mg, $9 \mathrm{~mol} \%)$ in deuterated chloroform $(0.7 \mathrm{~mL})$ at $50{ }^{\circ} \mathrm{C}$ was monitored by ${ }^{1} \mathrm{H}$ NMR spectroscopy until $48 \mathrm{~h}$ (Chart S-5). The reaction reached equilibrium within 48 h [2b:4b:(Z)-5b:(E)-5b = 1:5:18:76].

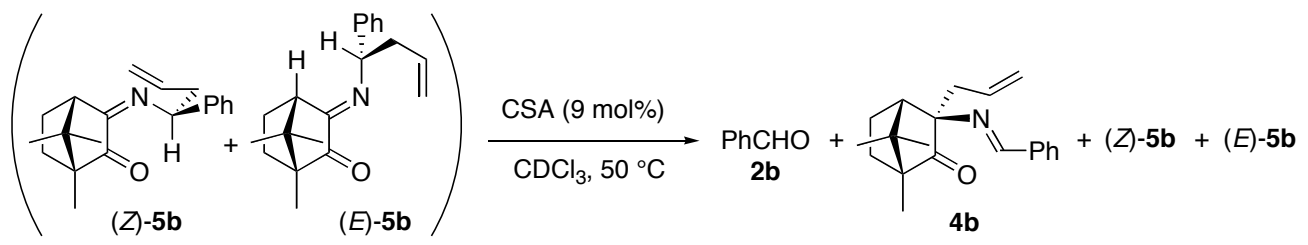

$1: 5: 18: 76$ 


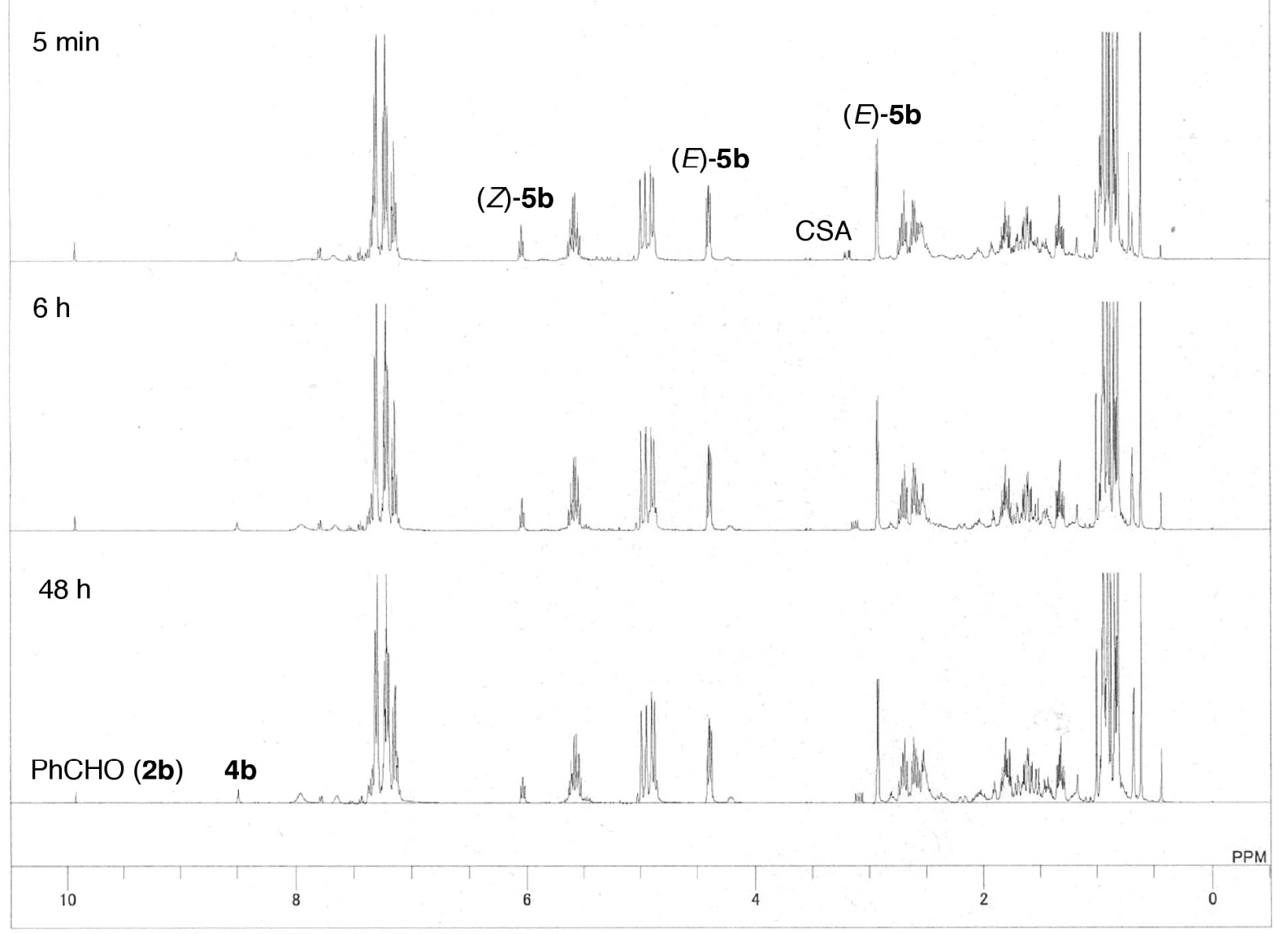

Chart S-5. NMR monitoring of reaction of $\mathbf{5 b}$

NMR analysis of reaction of 2-thiophenecarboxaldehyde (2f) with $1 \mathrm{a}$ in $\mathrm{CDCl}_{3}$

A mixture of chiral amine $\mathbf{1 a}(99.9 \mathrm{mg}, 0.48 \mathrm{mmol})$ and 2-thiophenecarboxaldehyde (2f) (55.2 mg, $0.49 \mathrm{mmol})$ in deuterated chloroform $(1.0 \mathrm{~mL})$ was stood for $24 \mathrm{~h}$ at $50{ }^{\circ} \mathrm{C}$ in a NMR tube. No rearranged product was observed at that stage. Then camphorsulfonic acid (11.8 $\mathrm{mg}, 11 \mathrm{~mol} \%$ ) was added to the mixture and the reaction was monitored by ${ }^{1} \mathrm{H}$ NMR spectroscopy at $50{ }^{\circ} \mathrm{C}$ until $48 \mathrm{~h}$ (Chart S-6). The reaction reached equilibrium within $48 \mathrm{~h}$ [2f:4f:(Z)-5f:(E)-5f = 4:35:18:43].

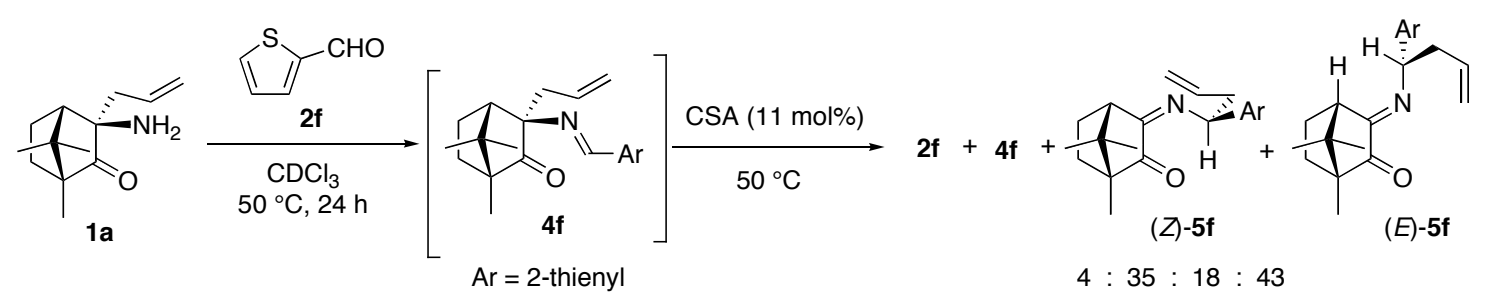




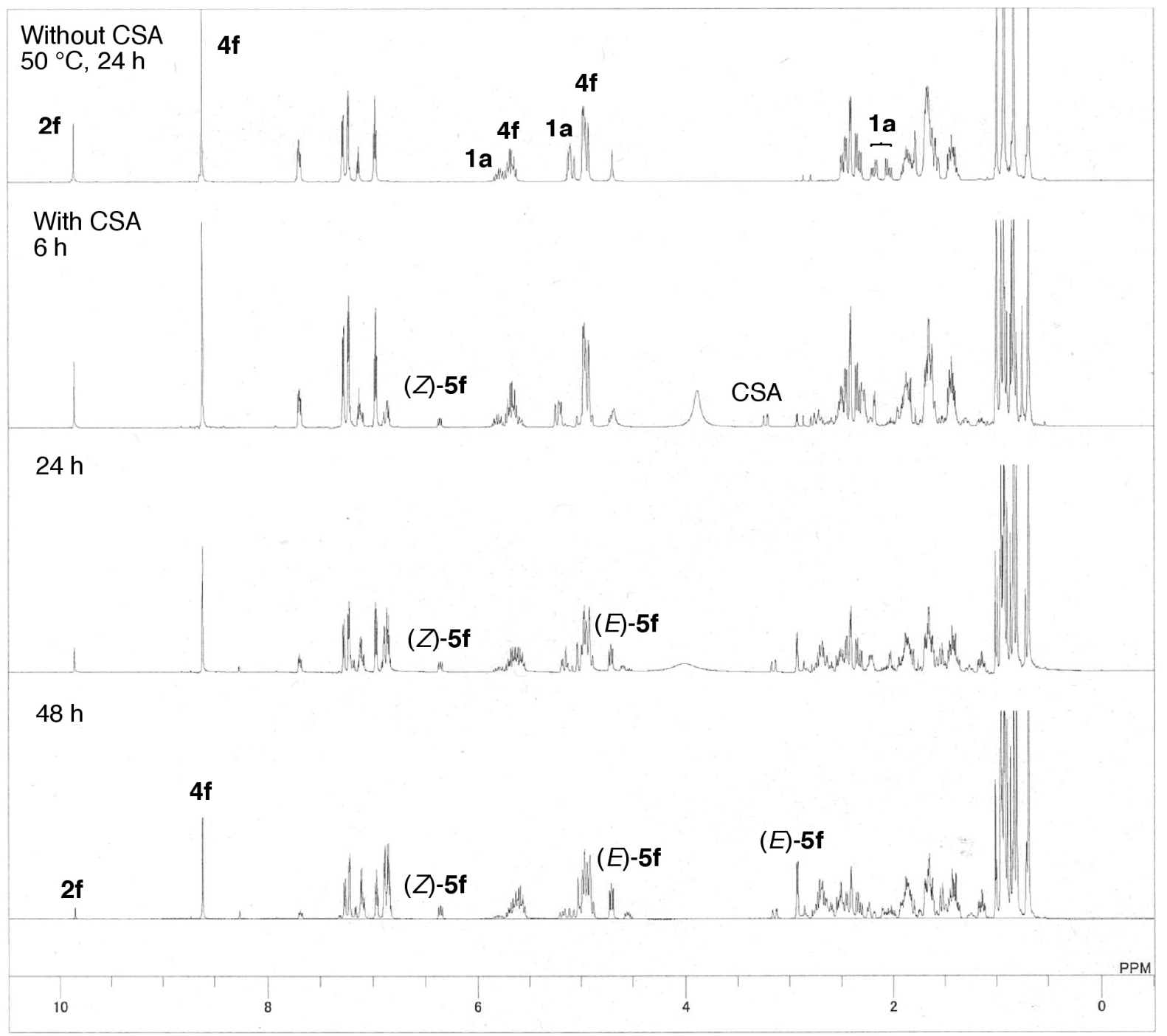

Chart S-6. NMR monitoring of reaction of $2 \mathbf{f}$ with 19

\section{References}

(1) Roush, W. R.; Walts, A. E. Tetrahedron Lett. 1985, 26, 3427.

(2) Sugiura, M.; Hirano, K.; Kobayashi, S. J. Am. Chem. Soc. 2004, 126, 7182.

(3) Chen, G.-M.; Ramachandran, P. V.; Brown, H. C. Angew. Chem. Int. Ed. 1999, 38, 825.

(4) Ramachandran, P. V.; Burghardt, T. E. Chem. Eur. J. 2005, 11, 4387.

(5) Felpin, F.-X.; Girard, S.; Vo-Thanh, G.; Robins, R. J.; Villiéras, J.; Lebreton, J. J. Org. Chem. 2001, 66, 6305.

(6) Kobayashi, S.; Ogawa, C.; Konishi, H.; Sugiura, M. J. Am. Chem. Soc. 2003, 125, 6610. 
C: $¥$ Documents and Settings $¥$ delta $¥$ My Documents $¥$ Personal Folder $¥$ Morichie $¥ \mathrm{~cm} 2 ¥ \mathrm{~cm} 2-180 ¥ \mathrm{~cm} 2-181$-pure-lit.als cm2-181-pure

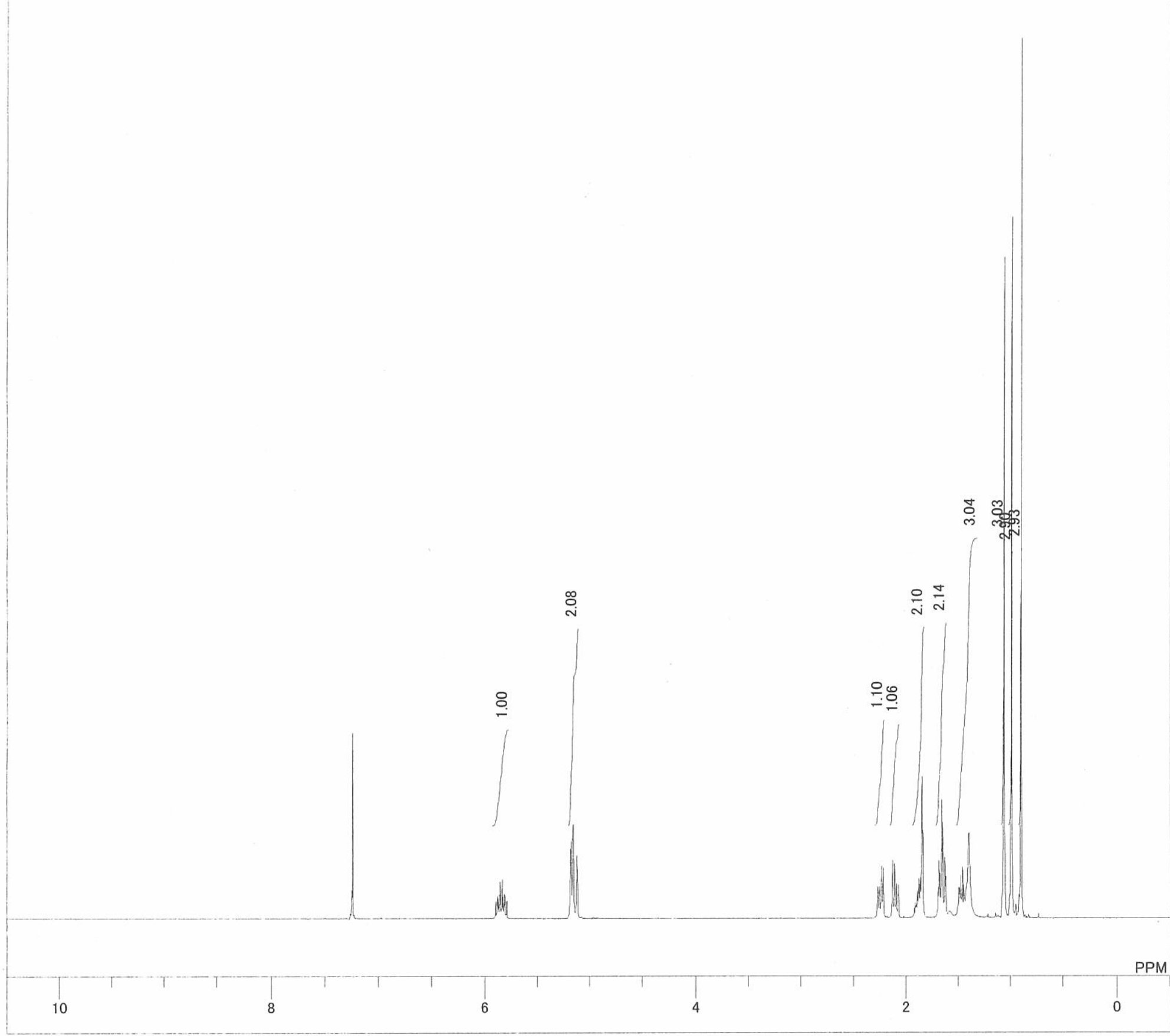

DFILE

COMNT

DATIM

OXNOC

OBFRQ

OBSET

POINT

FREQU

SCANS

ACQTM

$\mathrm{PD}$

PW1
IRNUC

IRNUC

SLVNT

EXREF

: Documents and Settings $¥$ delta $¥$ My Documents $¥$ cm2-181-pure

ingle_pulse.ex2 $399.78 \mathrm{MHz}$
$4.19 \mathrm{KHz}$ $4.19 \mathrm{KHz}$
$7.29 \mathrm{~Hz}$ 13107 $6002.31 \mathrm{~Hz}$ 8
$2.1837 \mathrm{sec}$ $2.0000 \mathrm{sec}$ 5.50 usec 7.24 $7.24 \mathrm{ppm}$ 34

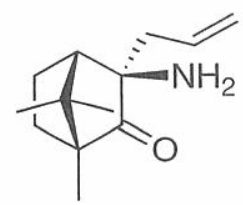

$1 a$ 
C: $¥$ Documents and Settings $¥$ delta $¥$ My Documents $¥$ Personal Folder $¥$ Morichie $¥ \mathrm{~cm} 2 ¥ \mathrm{~cm} 2-180 ¥ \mathrm{~cm} 2-181-$ pure $-13 \mathrm{C}$-lit.als cm2-181-pure-13C

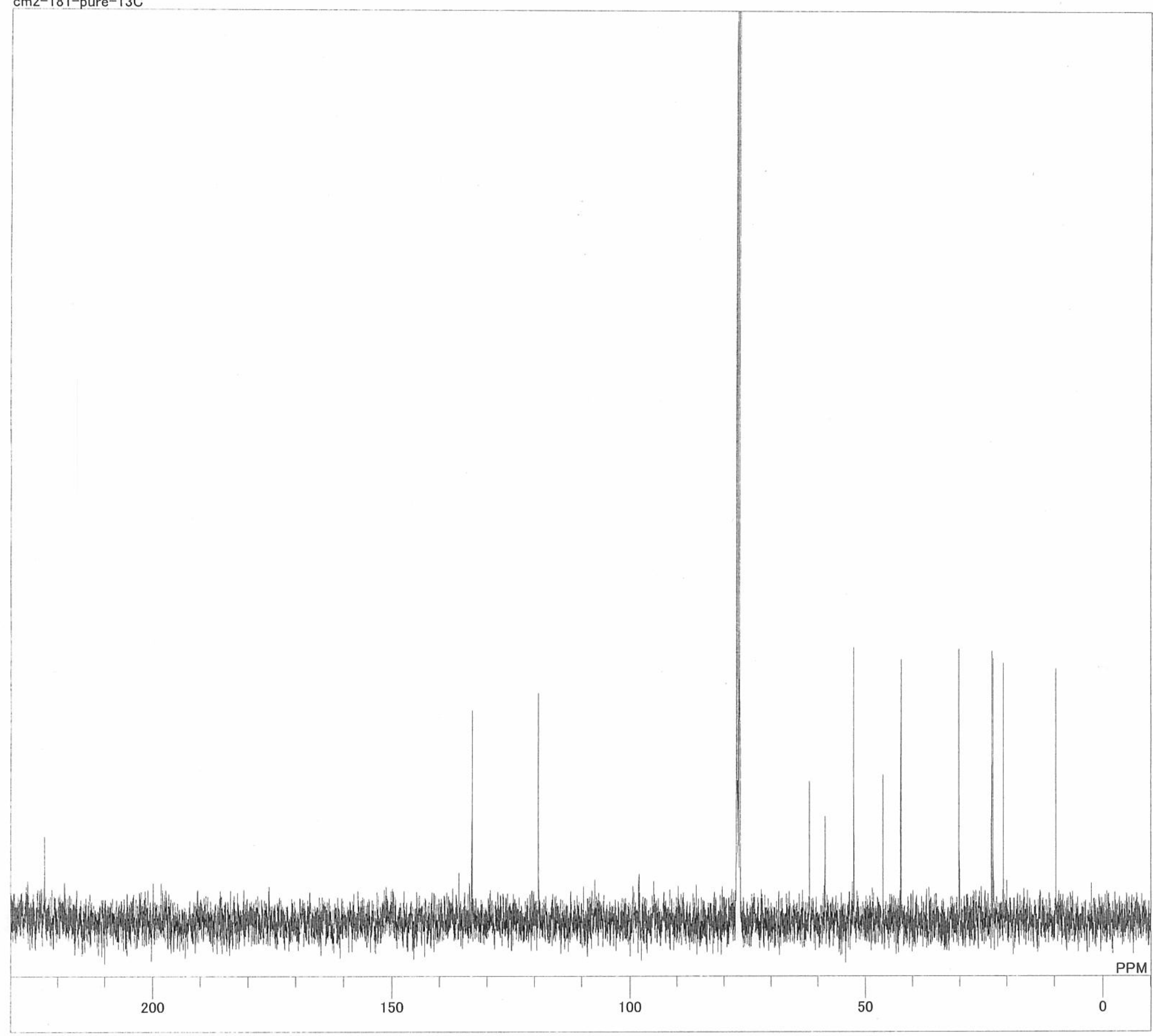

DFILE

COMNT

OBFRQ

OBSET

FREQU

SCANS

ACQTM

PD

PW1

RNUC

CTEMP

EXREF

RGAIN

C:¥Documents and Settings $¥$ delta $¥ M y$ Documents $¥$ cm2-181-pure-13C

$13 \mathrm{C}$

ulse_dec $100.53 \mathrm{MHz}$ $5.35 \mathrm{KHz}$
$5.86 \mathrm{~Hz}$ 32768 $31407.04 \mathrm{~Hz}$ 238
$1.0433 \mathrm{sec}$ $2.0000 \mathrm{sec}$ 3.33 usec 22.9 $77.00 \mathrm{ppm}$ $77.00 \mathrm{ppm}$
$0.12 \mathrm{~Hz}$ 50

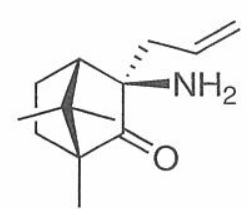

$1 a$ 
OBNUC $1 \mathrm{H}$

EXMOD single

OBFRQ

OBSET

$600.17 \mathrm{MHz}$

OBFIN

POINT

FREQU

SCANS

ACQTM

PD

PW1

IRNUC

CTEMP

SLVNT

EXREF

BF

RGAIN

$5.30 \mathrm{KHz}$

$5.47 \mathrm{~Hz}$

$5.47 \mathrm{~Hz}$
13107

$9008.87 \mathrm{~Hz}$

16

$1.4549 \mathrm{sec}$

$4.0000 \mathrm{sec}$

$1 \mathrm{H}$

7.30 usec

CDCL3

$19.6 \mathrm{c}$

$7.24 \mathrm{ppm}$

$0.12 \mathrm{~Hz}$

40

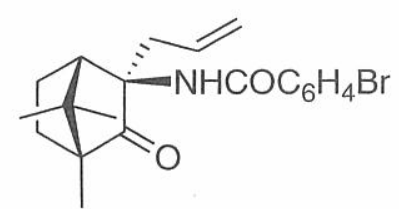

$p$-Bromobenzamide of $1 a$ 
DFILE

COMNT

DATIM

OBNUC

EXMOD

OBFRQ

OBSET

OBFIN

POINT

PREQU

FRQU

SCANS

ACQTM

PD

PW1

IRNUC

CTEMP

SLVNT

EXREF

BF

RGAIN
C.:Documents and Settings $¥$ All Users cm2-126

21-11-2005 19:56:13

$13 \mathrm{C}$

single_pulse_dec

$150.92 \mathrm{MHz}$

$8.52 \mathrm{KHz}$

$1.74 \mathrm{~Hz}$

32768

$47348.48 \mathrm{~Hz}$

$0.6921 \mathrm{sec}$

$2.0000 \mathrm{sec}$

$1 \mathrm{H}$

20.1 c

CDCL3

$77.00 \mathrm{ppm}$

$0.12 \mathrm{~Hz}$

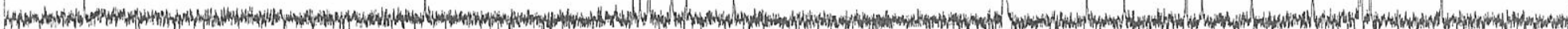

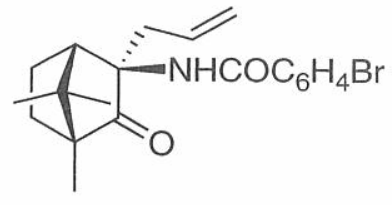

p-Bromobenzamide of $1 \mathrm{a}$ 
DBNUC

EXMOD

OBFRQ

OBFIN

POINT

FREQU

SCANS

ACQTM

PD

PW1

IRNUC

CTEMP
SLVNT

SLVNT
EXREF

BF

7-04-2006 13:35:22

ingle_pulse.ex2 $399.78 \mathrm{MHz}$
$4.19 \mathrm{KHz}$ $7.29 \mathrm{~Hz}$ 16384

$7503.00 \mathrm{~Hz}$

8

$2.1837 \mathrm{sec}$
$2.0000 \mathrm{sec}$

5.50 usec

$7.24 \mathrm{ppm}$
$0.12 \mathrm{~Hz}$
34

$0.12 \mathrm{~Hz}$

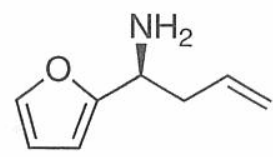

$3 g$

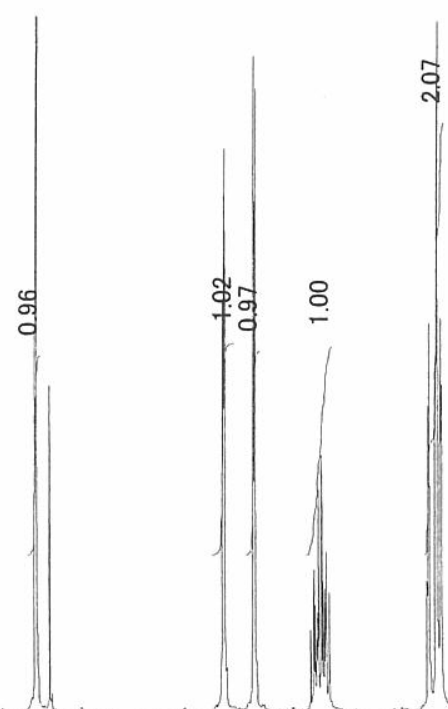


C: $¥$ Documents and Settings $¥$ delta $¥ M y$ Documents $¥$ Personal Folder $¥$ Morichie $¥ \mathrm{~cm} 3-40 ¥ \mathrm{~cm} 3-40-\mathrm{OH}-$ pure $-13 \mathrm{C}$.als cm3-40-OH-pure-13C

OBNUC

OBFRQ

OBFRQ

OBSET

POINT

FREQU

SCANS

ACQTM

PD

PW1

IRNUC

CTEMP

SLVNT

$\mathrm{BF}$

RGAIN

: $¥$ Documents and Settings $¥$ delta $¥$ My Documents $¥$ cm3-40-OH-pure-13C

single_pulse_dec

$100.53 \mathrm{MHz}$
$5.35 \mathrm{KHz}$

$5.86 \mathrm{~Hz}$

32768

$31407.04 \mathrm{~Hz}$

184

$1.0433 \mathrm{sec}$
$2.0000 \mathrm{sec}$

3.33 usec

21.9

$77.00 \mathrm{ppm}$

50

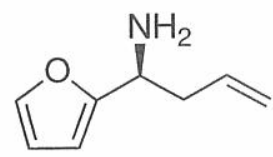

$3 g$ 
C: $¥$ Documents and Settings $¥$ delta $¥$ My Documents $¥$ Personal Folder $¥$ Morichie $¥ \mathrm{~cm} 3-20 ¥ \mathrm{~cm} 3-26-1 \mathrm{H}-$ lit.als $\mathrm{cm} 3-28-1 \mathrm{H}$

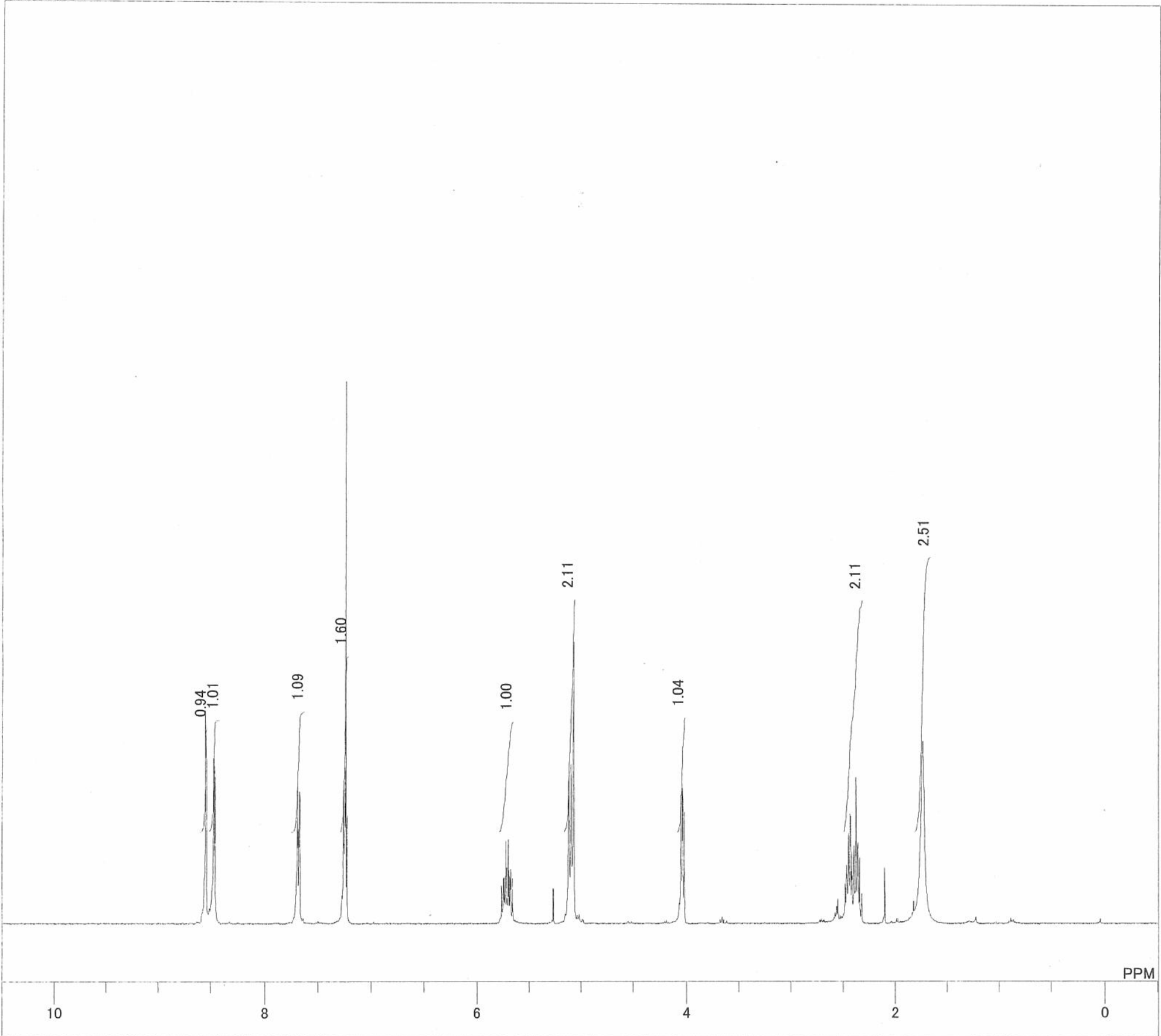

DFILE

COMNT

DATIM

DANUC

OBFRQ

OBFRQ

OBSET

POINT

FREQU

SCANS

ACQTM

PD

PW1

RNUC

CTEMP

EXREF

BF

Documents and Settings $¥$ delta $¥$ My Documents $¥$ m3-28-1H 14:51:06

399.78 $\mathrm{MHz}$ $399.78 \mathrm{MHz}$
$4.19 \mathrm{KHz}$ $4.19 \mathrm{KHz}$
$7.29 \mathrm{~Hz}$

13107

$6002.31 \mathrm{~Hz}$

8
$.1837 \mathrm{sec}$

$2.0000 \mathrm{sec}$ 5.50 usec

23.6

$7.24 \mathrm{ppm}$

38

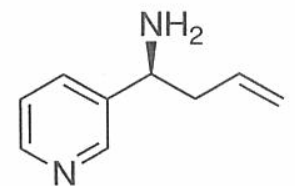

3h 
C: $¥$ Documents and Settings $¥$ delta $¥$ My Documents $¥$ Personal Folder $¥$ Morichie $¥ \mathrm{~cm} 3-20 ¥ \mathrm{~cm} 3-28-13 \mathrm{C}$.als

1

DFILE

COMNT

COMNT

EXMOD

EXMOD

OBFRQ

OBFIN

POINT

FREQU

SCANS

ACQTM

PD

PW1

IRNUC

CTEMP

EXREF

RGAIN

: $:$ Documents and Settings $¥$ delta $¥$ My Documents $¥$ (13C

gle_pulse_dec

$100.53 \mathrm{MHz}$
$5.35 \mathrm{KHz}$

$5.86 \mathrm{~Hz}$

$31407.04 \mathrm{~Hz}$

200

$1.0433 \mathrm{sec}$

$2.0000 \mathrm{sec}$

$1 \mathrm{H}$

24.0

$77.00 \mathrm{ppm}$

54

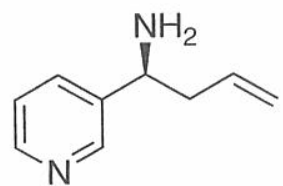

3h 


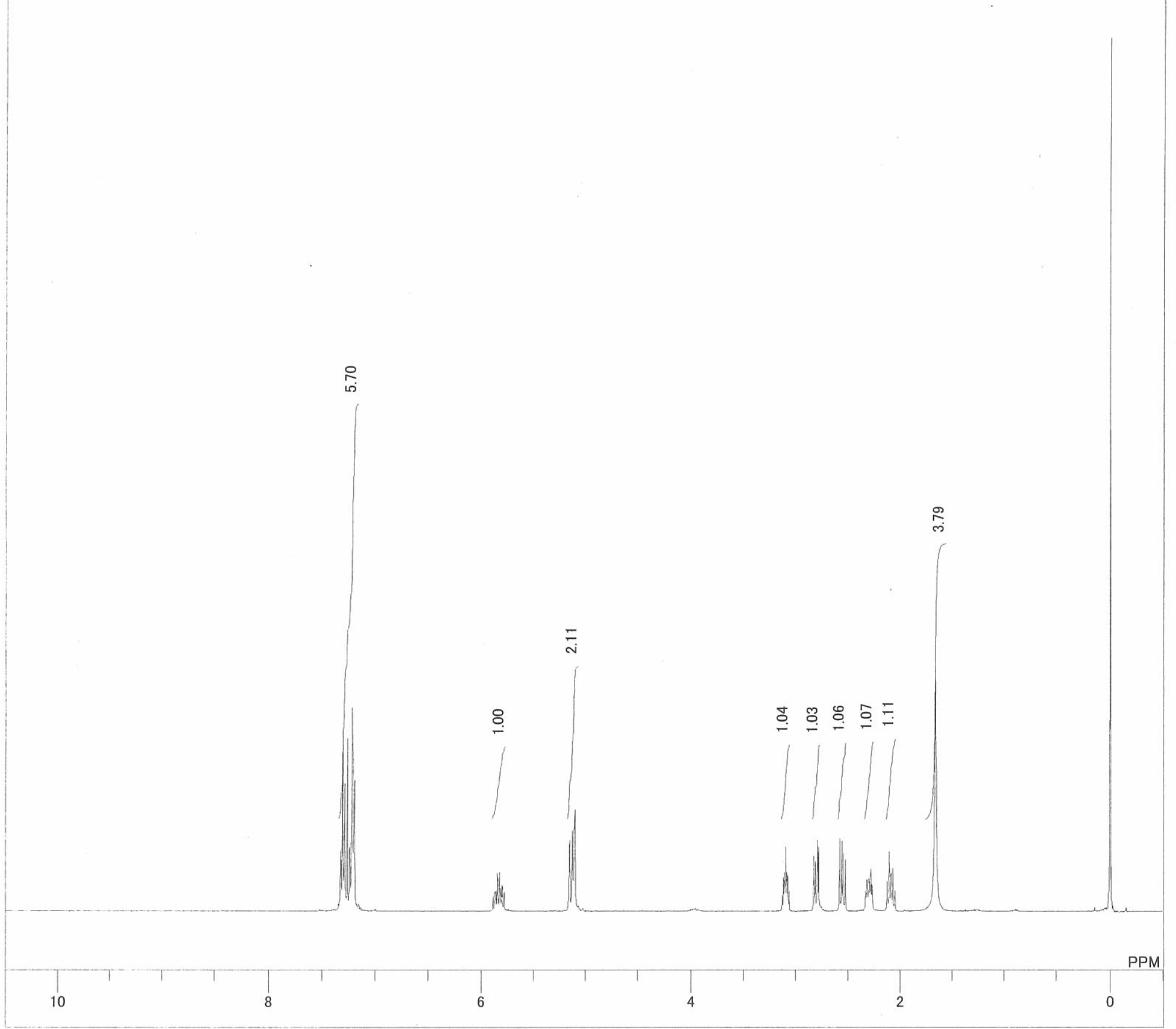

C:¥Documents and Settings $¥$ delta $¥ M y$ Documents $¥$

EXMOD

OBFRQ

OBFIN

POINT

(14:00:16

EQU

SCANS

ACQTM

PD

PW1

PWN

CINT

EXREF

RGAIN

$399.78 \mathrm{MHz}$

$7.19 \mathrm{~Hz}$

13107

$6002.31 \mathrm{~Hz}$

8

$2.1837 \mathrm{sec}$
$2.0000 \mathrm{sec}$

5.50 usec

$24.2 \mathrm{c}$

$0.00 \mathrm{ppm}$

$0.12 \mathrm{~Hz}$

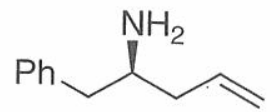

3j 
C: $¥$ Documents and Settings $¥$ delta $¥ M y$ Documents $¥$ Personal Folder $¥$ Morichie $¥ \mathrm{~cm} 3-80 ¥ \mathrm{~cm} 3-84-13$ C.als

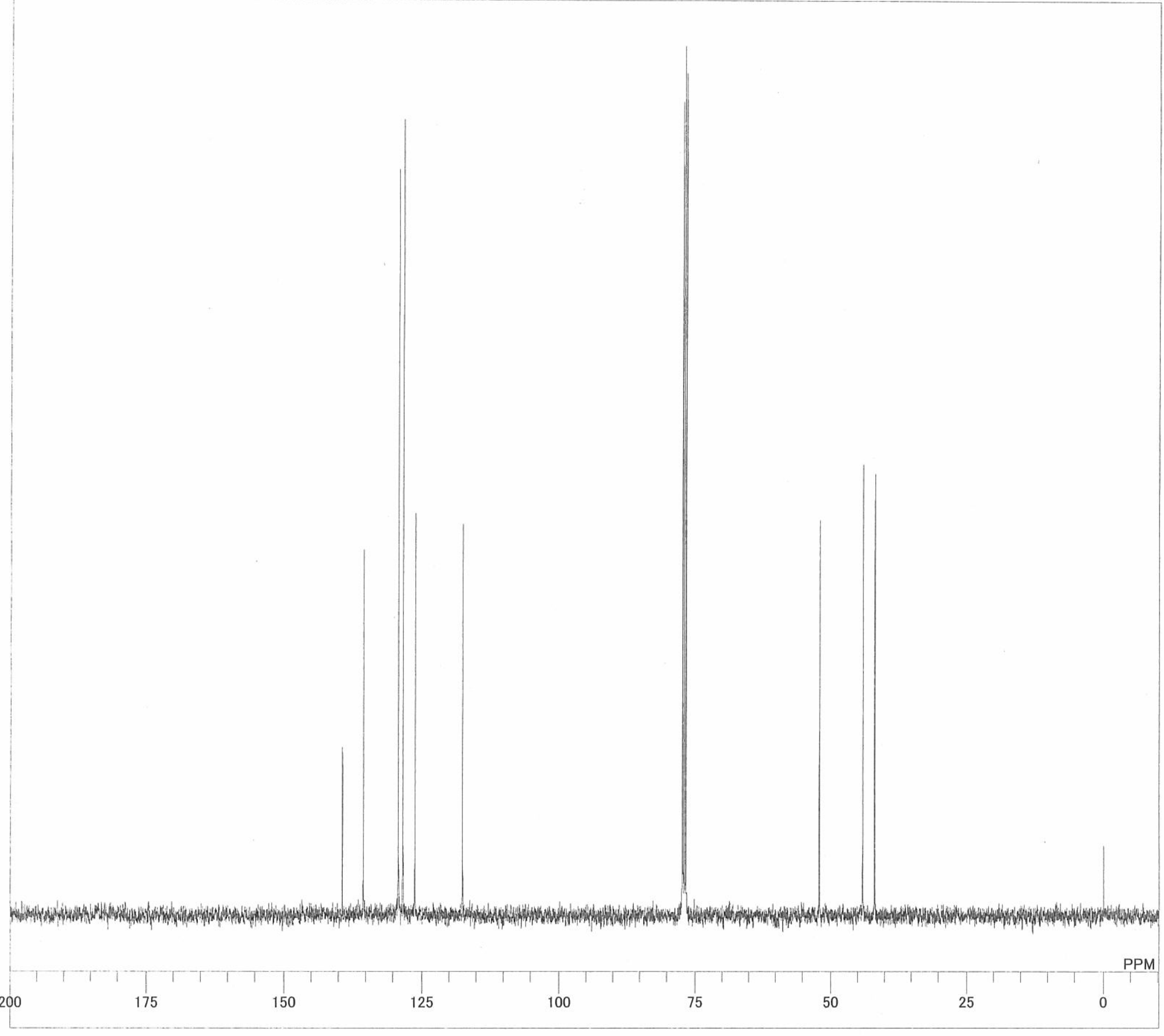

OBNUC

OBFRQ
OBSET

OBFIN

POINT

FREQU

SCANS

ACQTM

PD

PW1

CTEMP

STENT

BF

RGAIN

: $¥$ Documents and Settings $¥$ delta $¥$ My Documents $¥$

$13 \mathrm{C}$
single pulse dec

$100.53 \mathrm{MHz}$

$5.35 \mathrm{KHz}$

32768

200

$2.0000 \mathrm{sec}$

3.33 usec

24.8

$0.00 \mathrm{ppm}$

$0.12 \mathrm{~Hz}$

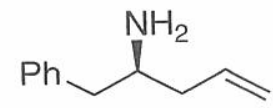

3j 
C: $¥ D$ Documents and Settings $¥$ delta $¥ \mathrm{My}$ Documents $¥ \mathrm{P}$ ersonal Folder $¥$ Morichie $¥ \mathrm{~cm} 3-40 ¥ \mathrm{~cm} 3-43-\mathrm{OH}$-pure.als

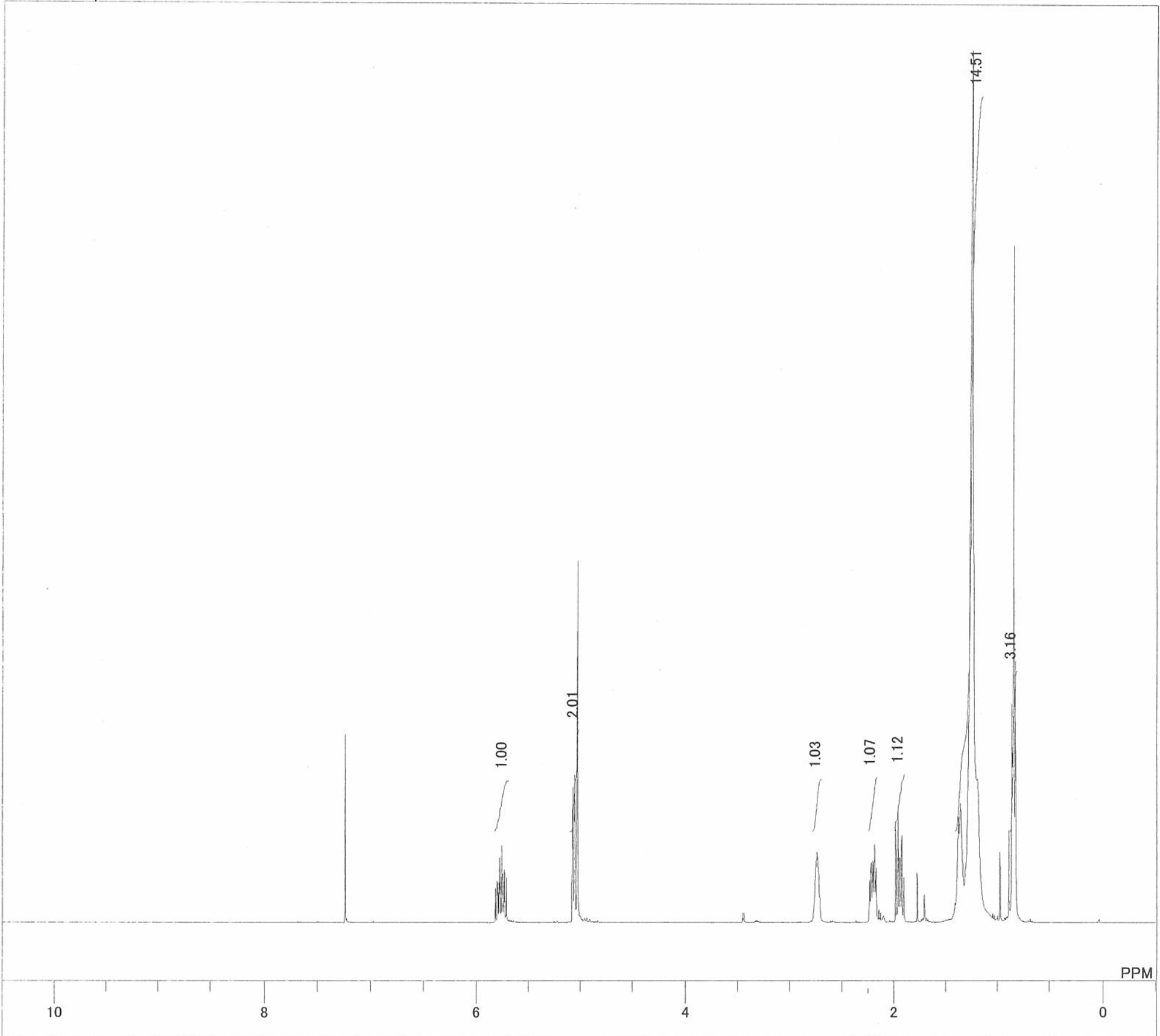

DATIM

OBNUC

EXMOD

OBFRQ

OBFIN

POINT

FREQU

SCANS

ACQTM

PD
PW1

RNUC

CTEMP

SLVNT

EXREF

RGAIN

:¥Documents and Settings $¥$ delta $¥$ My Documents (10)

(

$399.78 \mathrm{MHz}$
single_pulse.ex2 $4.19 \mathrm{KHz}$ $7.29 \mathrm{~Hz}$

13107

$6002.31 \mathrm{~Hz}$

8
$2.1837 \mathrm{sec}$

$2.0000 \mathrm{sec}$

5.50 usec

$22.5 \mathrm{c}$

$7.24 \mathrm{ppm}$

$0.12 \mathrm{H}$
34

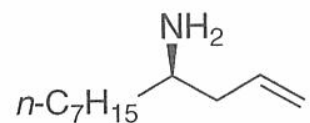

3k 
C: $¥$ Documents and Settings $¥$ delta $¥ \mathrm{My}$ Documents $¥ \mathrm{P}$ ersonal Folder $¥$ Morichie $¥ \mathrm{~cm} 3-40 ¥ \mathrm{~cm} 3-43-\mathrm{OH}-$ pure $-13 \mathrm{C}$.als cm3-43-OH-pure-13C

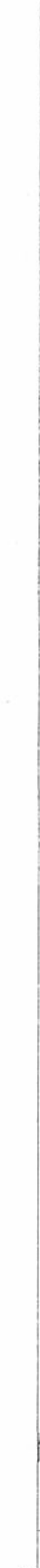

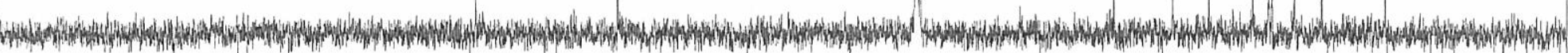

\section{DFILE}

COMNT

DATIM

OBNUC

EXMOD

OBFRQ

OBSET

POINT

FREQU

SCANS

ACQTM

PD

PW1
IRNUC

CTEMP
SLVNT
EXREF

BF
RGAIN

C: $¥$ Documents and Settings $¥$ delta $¥$ My Documents $¥$ cm3-43-OH-pure-13C

gle_pulse_dec $100.53 \mathrm{MHz}$
$5.35 \mathrm{KHz}$ $5.35 \mathrm{KHz}$
$5.86 \mathrm{~Hz}$ 32768 $31407.04 \mathrm{~Hz}$ 156 $1.0433 \mathrm{sec}$
$2.0000 \mathrm{sec}$ 3.33 usec 1H 22.9 DCL3 $77.00 \mathrm{ppm}$
$0.12 \mathrm{~Hz}$

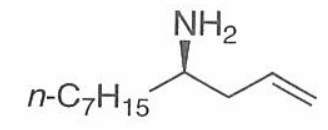

3k 
C: $¥$ Documents and Settings $¥$ delta $¥$ My Documents $¥$ Personal Folder $¥$ Morichie $¥ \mathrm{~cm} 3-40 ¥ \mathrm{~cm} 3-43-B z$.als

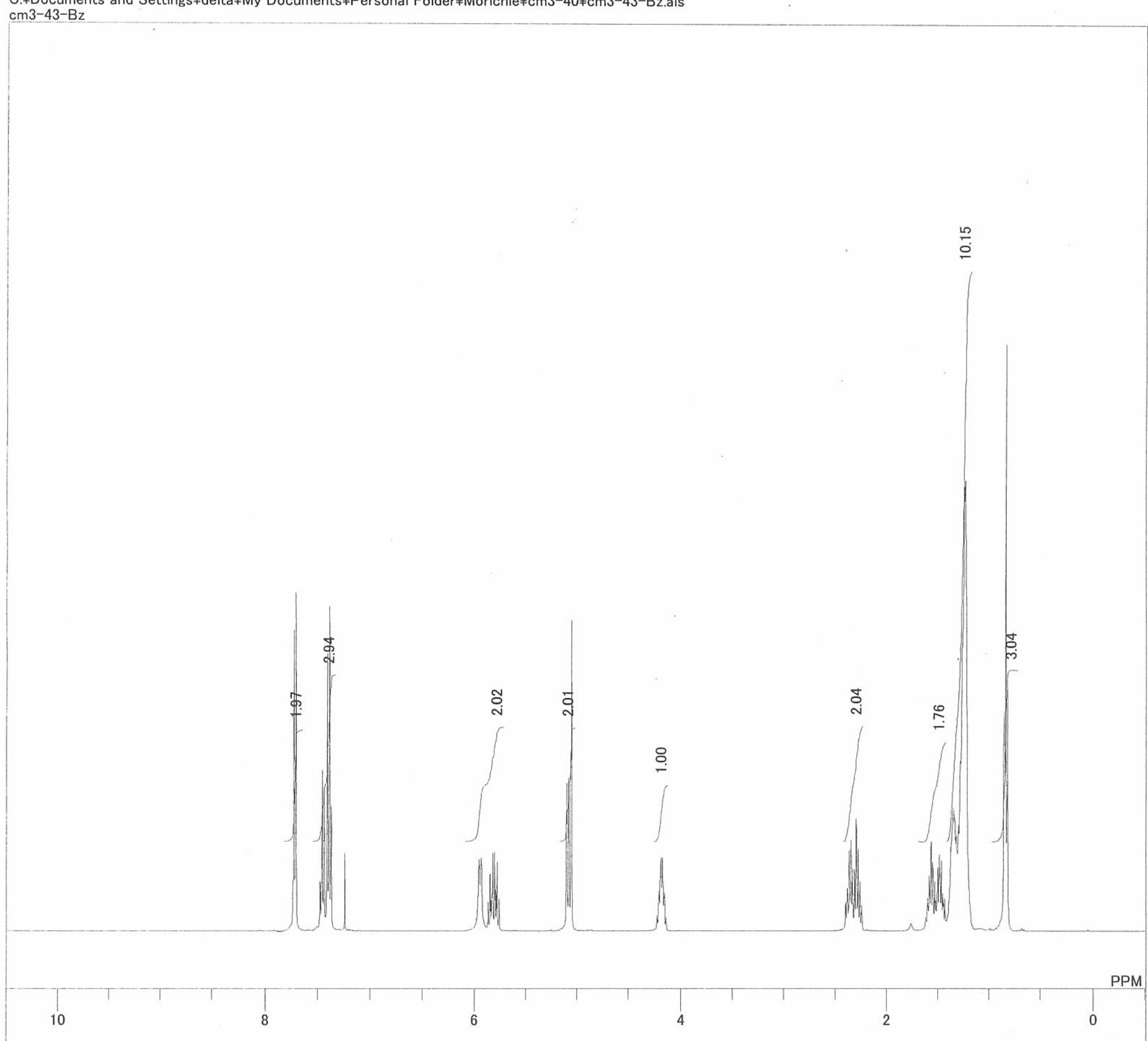

DFILE

COMNT

DATIM

OBNUC

OXMOD

OBFRQ

OBSEIN

POINT

FREQU

SCANS

ACQTM
PW1

PW1

RNUC

CTEMP

SLVNT

BF

RGAIN

: $¥$ Documents and Settings $¥$ delta $¥$ My Documents $¥$

$399.78 \mathrm{MHz}$ $399.78 \mathrm{MHz}$
$4.19 \mathrm{KHz}$ $4.19 \mathrm{KHz}$
$7.29 \mathrm{~Hz}$

13107

$6002.31 \mathrm{~Hz}$

$2.1837 \mathrm{sec}$

$2.0000 \mathrm{sec}$

5.50 usec

23.2

$7.24 \mathrm{ppm}$

28

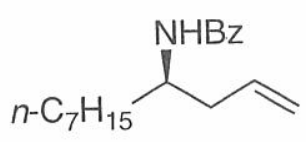

Benzamide of $\mathbf{3 k}$ 
C: $¥ D$ Documents and Settings $¥$ delta $¥$ My Documents $¥$ Personal Folder $¥$ Morichie $¥ \mathrm{~cm} 3-40 ¥ \mathrm{~cm} 3-43-B z-13 \mathrm{C}$.als

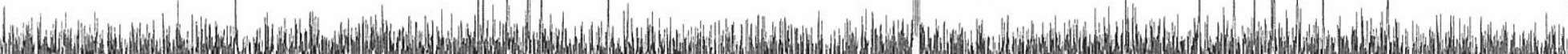

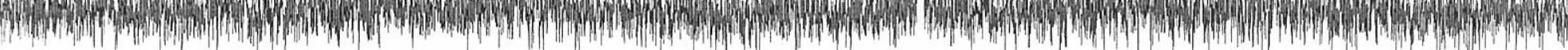

DATIM

EXMOD

EXMOD

OBFRQ

OBFIN

POINT

FREQU

SCANS

ACQTM

PD

PW1

IRNUC

SLVNT

BF

Documents and Settings $¥$ delta $¥ M y$ Documents $\mathrm{cm} 3-43-\mathrm{Bz}-13 \mathrm{C}$

$13 \mathrm{C}$

single_pulse_dec $5.35 \mathrm{KHz}$ $5.86 \mathrm{~Hz}$ 32768 $31407.04 \mathrm{~Hz}$ 38 $1.0433 \mathrm{sec}$ $2.0000 \mathrm{sec}$ $2.000 \mathrm{sec}$
$3.33 \mathrm{usec}$ $23.5 \mathrm{c}$ $77.00 \mathrm{ppm}$ $77.00 \mathrm{ppm}$
$0.12 \mathrm{~Hz}$

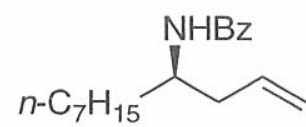

Benzamide of $\mathbf{3 k}$ 
C: $¥ D o c u m e n t s$ and Settings $¥ A l l$ Users $¥$ Documents $¥$ Morichie $¥ \mathrm{~cm} 3-124-0$ PROTON.als $\mathrm{cm} 3-124-0$

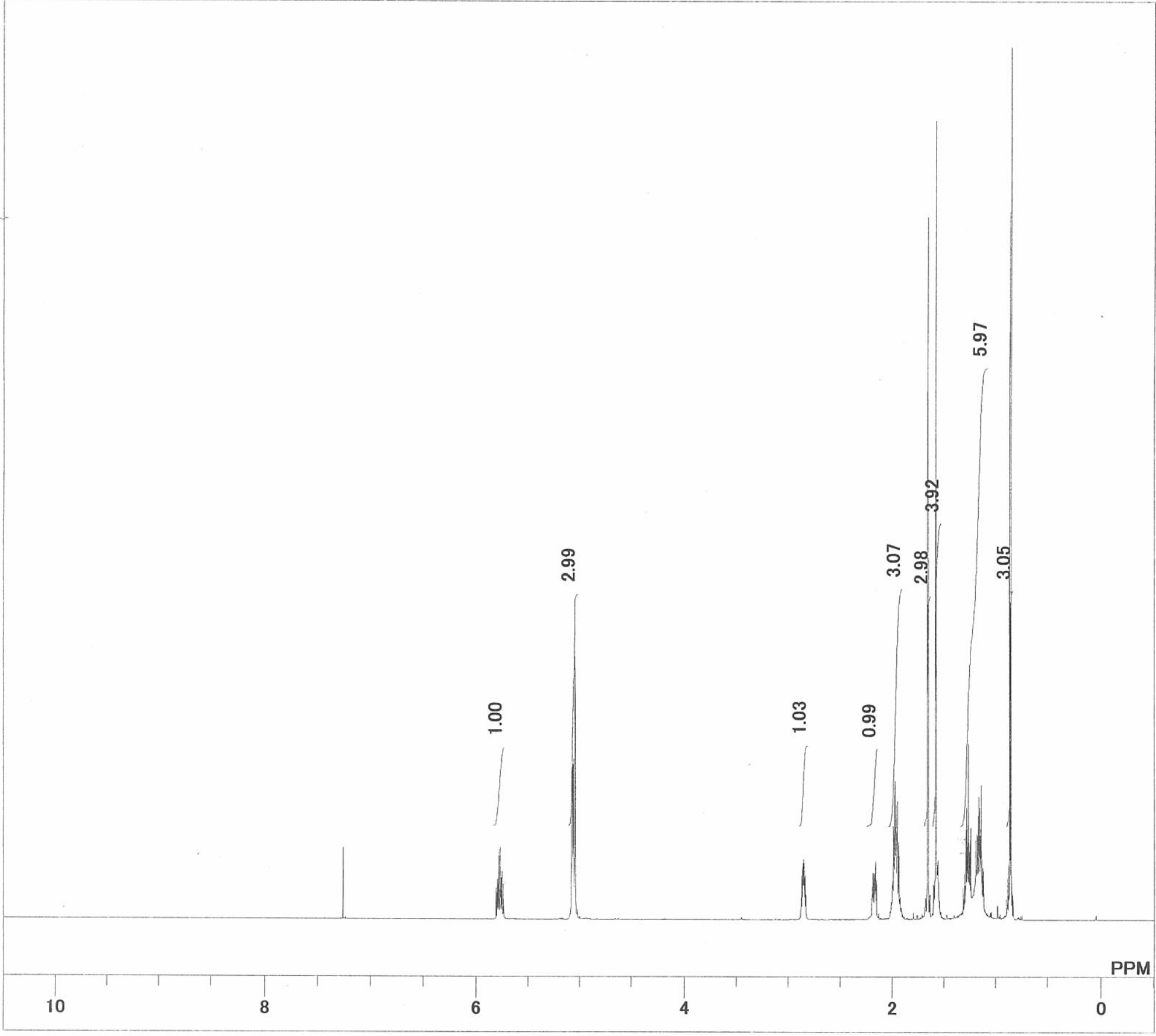

DFILE

COMNT

DATIM

OBNUC

EXMOD

OBFRQ

OBSET

OBFIN

POINT

PREQU

TRQ

SCANS

ACQTM

PD

PW1

IRNUC

CTEMP

SLVNT

EXREF

BF

RGAIN
.:Documents and Settings $¥$ All Users cm3-124-0

07-06-2006 13.28.24

$1 \mathrm{H}$

single_pulse.ex2 $600.17 \mathrm{MHz}$ $5.30 \mathrm{KHz}$

$5.47 \mathrm{~Hz}$

13107

$9008.87 \mathrm{~Hz}$

16

16

$1.4549 \mathrm{sec}$

$4.0000 \mathrm{sec}$

7.30 usec

$1 \mathrm{H}$

$21.6 \mathrm{c}$

CDCL3

21.6

$7.26 \mathrm{ppm}$

32

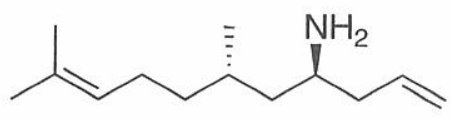

(4S,6S)-3I

PPM 
C:¥Documents and Settings $¥ A l l$ Users¥Documents $¥$ Morichie $¥ \mathrm{~cm} 3-124-0$ CARBON.als cm3-124-0

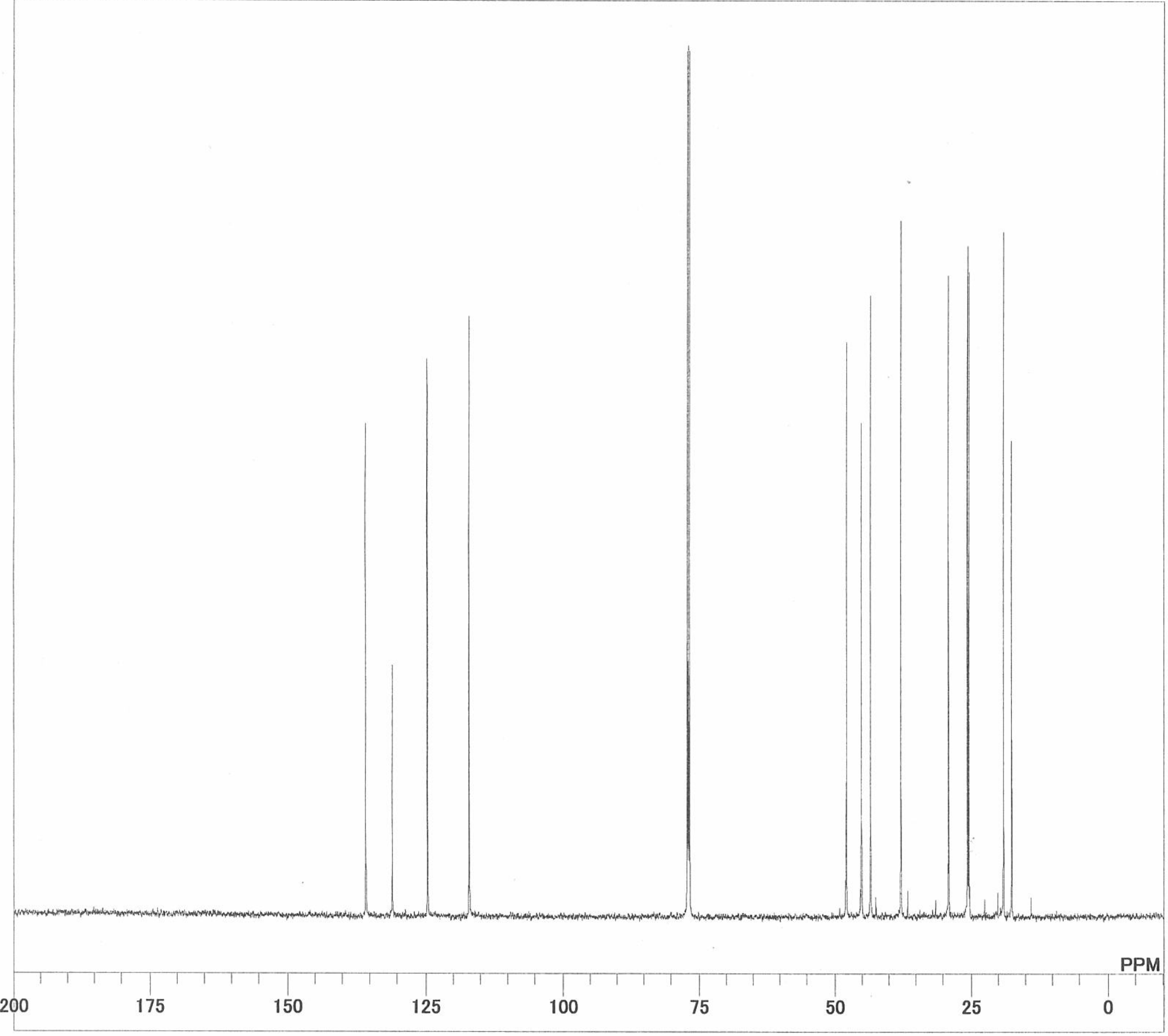

DFILE

COMNT

DATIM

OBNUC

EXMOD

OBFRQ

OBSET

OBFIN

POINT

FREQU

SCANS

ACQTM

PD

PW1

IRNUC

CTEMP

SLVNT

EXREF

BF

RGAIN

C:¥Documents and Settings $¥ A l l$ Users cm3-124-0

07-06-2006 14:15:06

$13 \mathrm{C}$

single_pulse_dec

$150.92 \mathrm{MHz}$

$8.52 \mathrm{KHz}$

$1.74 \mathrm{~Hz}$

$47348.48 \mathrm{~Hz}$

1024

$0.6921 \mathrm{sec}$

$2.0000 \mathrm{sec}$

$1 \mathrm{H}$

usec

22.1

3

77.00 ppm

$0.12 \mathrm{~Hz}$

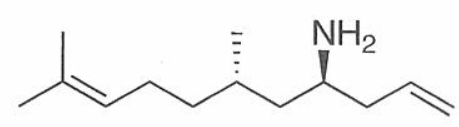

(4S,6S)-3I 
DFILE

COMNT

DATIM

OBNUC

EXMOD

OBSET

OBFIN

POINT

FREQU

FREQU

SCANS

ACQTM

PD

PW1

IRNUC

CTEMP

SLVNT

EXREF

BF

RGAIN

C: ¥Documents and Settings¥All Users cm3-126

08-06-2006 11:46:06

$1 \mathrm{H}$

single_pulse.ex2 $600.17 \mathrm{MHz}$ $5.30 \mathrm{KHz}$

$5.47 \mathrm{~Hz}$

13107

$9008.87 \mathrm{~Hz}$

16

$1.4549 \mathrm{sec}$

$4.0000 \mathrm{sec}$

7.30 usec

$1 \mathrm{H}$

$21.4 \mathrm{c}$

CDCL3

$7.26 \mathrm{ppm}$

$0.12 \mathrm{~Hz}$

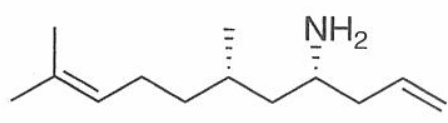

(4R,6S)-3|

PPM 
DFILE

COMNT

DATIM

DATIM

OBNUC

EXMOD

OBSET

OBFIN

POINT

FREQU

SCANS

ACQTM

PD

PW1

IRNUC

CTEMP

SLVNT

EXREF

EXRE

RGAIN
C:¥Documents and Settings $¥ A$ Il Users $¥$ cm3-126

08-06-2006 12:32:48

$13 \mathrm{C}$

single_pulse dec

$150.92 \mathrm{MHz}$ $8.52 \mathrm{KHz}$

$1.74 \mathrm{~Hz}$

32768

$47348.48 \mathrm{~Hz}$

1024

$0.6921 \mathrm{sec}$

$2.0000 \mathrm{sec}$

$1 \mathrm{H}$

usec

CDCL3

$77.00 \mathrm{ppm}$

$0.12 \mathrm{~Hz}$

60

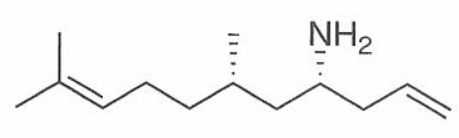

$(4 R, 6 S)-3 \mid$ 
C: ¥Documents and Settings $¥$ delta $¥$ My Documents $¥$ Personal Folder $¥$ Morichie $¥ \mathrm{~cm} 3-50 ¥ \mathrm{~cm} 3-53-$ Bz.als

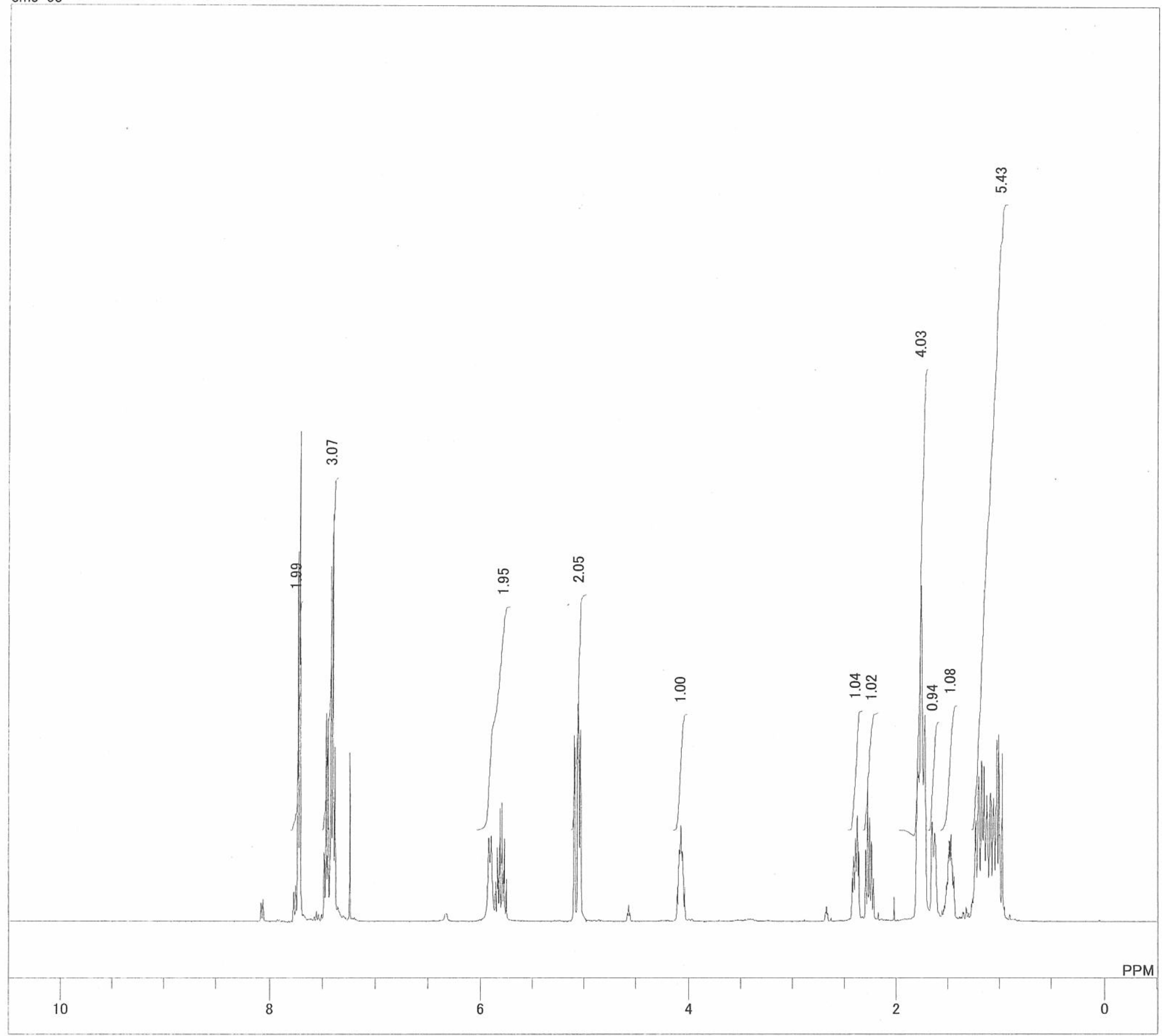

DFILE

COMNT

COMN

EXMOD

EXMFD

OBFRQ

OBFIN

POINT

FREQU

SCANS

ACQTM

PD

PW1

PWI

TEMP

RGAIN

₹Documents and Settings $¥$ delta $¥$ My Documents $¥$ $11-04-200$

-04-2006 19:38:25

$399.78 \mathrm{MHz}$

$399.78 \mathrm{MHz}$
$4.19 \mathrm{KHz}$

$7.29 \mathrm{~Hz}$

13107

$6002.31 \mathrm{~Hz}$

8
$2.1837 \mathrm{sec}$

$2.0000 \mathrm{sec}$

5.50 usec

22.7

$7.24 \mathrm{ppm}$

$0.12 \mathrm{~Hz}$

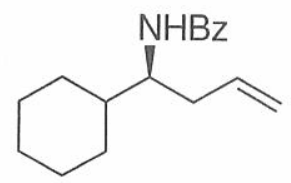

Benzamide of $3 \mathrm{~m}$ 
C: $¥$ Documents and Settings $¥$ delta $¥$ My Documents $¥$ Personal Folder $¥$ Morichie $¥ \mathrm{~cm} 3-50 ¥ \mathrm{~cm} 3-53-B z-13 \mathrm{C}$.als

\section{EXMOD}

OBFRQ

OBSET

OBFIN

FREQU

SCANS

ACQTM

PD

PW1

IRNUC

CTEMP
SLVNT

EXREF

RGAIN

:¥Documents and Settings $¥$ delta $¥$ My Documents $¥$ cm3-53-13c

11-04-2006 19:41:06

$100.53 \mathrm{MHz}$ $5.35 \mathrm{KHz}$ $5.86 \mathrm{~Hz}$ 32768 $31407.04 \mathrm{~Hz}$

39 $1.0433 \mathrm{sec}$
$2.0000 \mathrm{sec}$
$3.33 \mathrm{usec}$ 3.33 usec

$1 \mathrm{H}$ $77.00 \mathrm{ppm}$ $0.12 \mathrm{~Hz}$
50

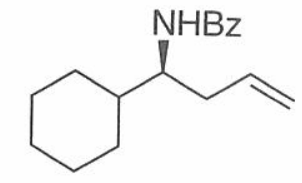

Benzamide of $3 \mathrm{~m}$ 
C: $¥$ Documents and Settings $¥$ delta $¥$ My Documents $¥$ Personal Folder $¥$ Morichie $¥ \mathrm{~cm} 3-40 ¥ \mathrm{~cm} 3-48-$ pure-lit.als

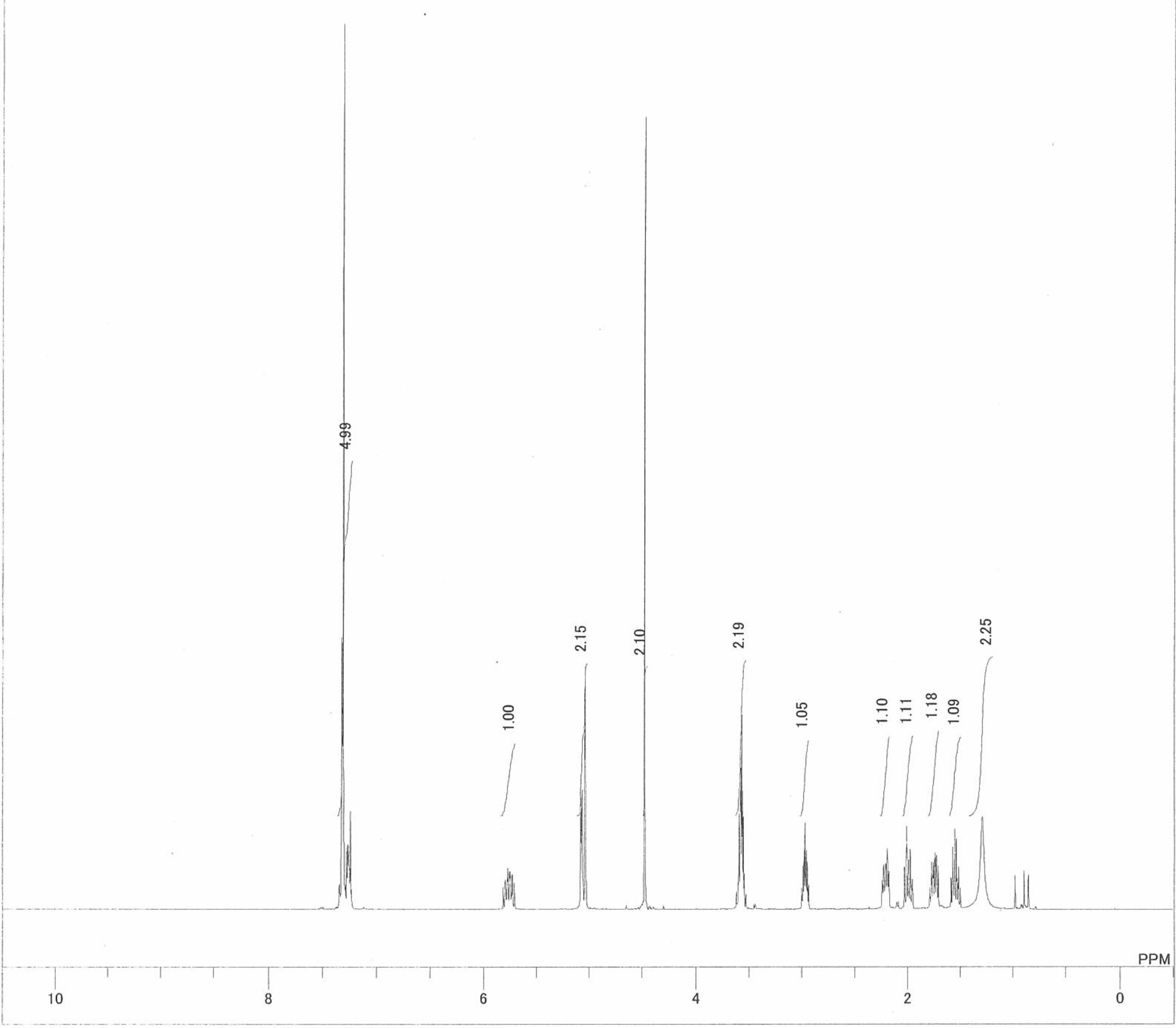

DFILE

COMNT

DATIM

DXNUC

BFRQ

BSET

BBFIN

POINT

SCANS

ACQTM

PDI

IRNUC 1

TEMP

RGAIN

7-04-2006 15:28:

gle_pulse.ex2
$399.78 \mathrm{MHz}$

$4.19 \mathrm{KHz}$

$7.29 \mathrm{~Hz}$

13107

$6002.31 \mathrm{~Hz}$

8

$2.1837 \mathrm{sec}$

$5.50 \mathrm{sec}$

CDCL3

$7.24 \mathrm{ppm}$
$0.12 \mathrm{~Hz}$

32

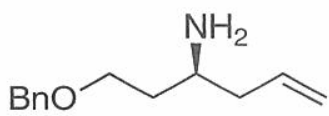

$3 n$ 
DFILE

COMNT

DATIM

OBNUC

OBNUC

OBFRQ

OBSET

OBFIN

POINT

SCANS

SCANS

PD

PW1

IRNUC

CTEMP
SLVNT

EXREF

EXREF

RGAIN

CDCL

$77.00 \mathrm{ppm}$

$0.12 \mathrm{~Hz}$

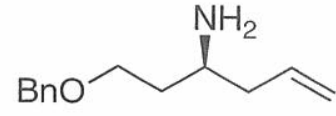

$3 n$

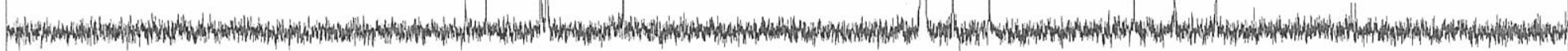


C: $¥$ Documents and Settings $¥$ delta $¥$ My Documents $¥$ Personal Folder $¥$ Morichie $¥ \mathrm{~cm} 3-30 ¥ \mathrm{~cm} 3-31-\mathrm{OH}-$ pure $1-$ lit.als cm3-31-OH-pure

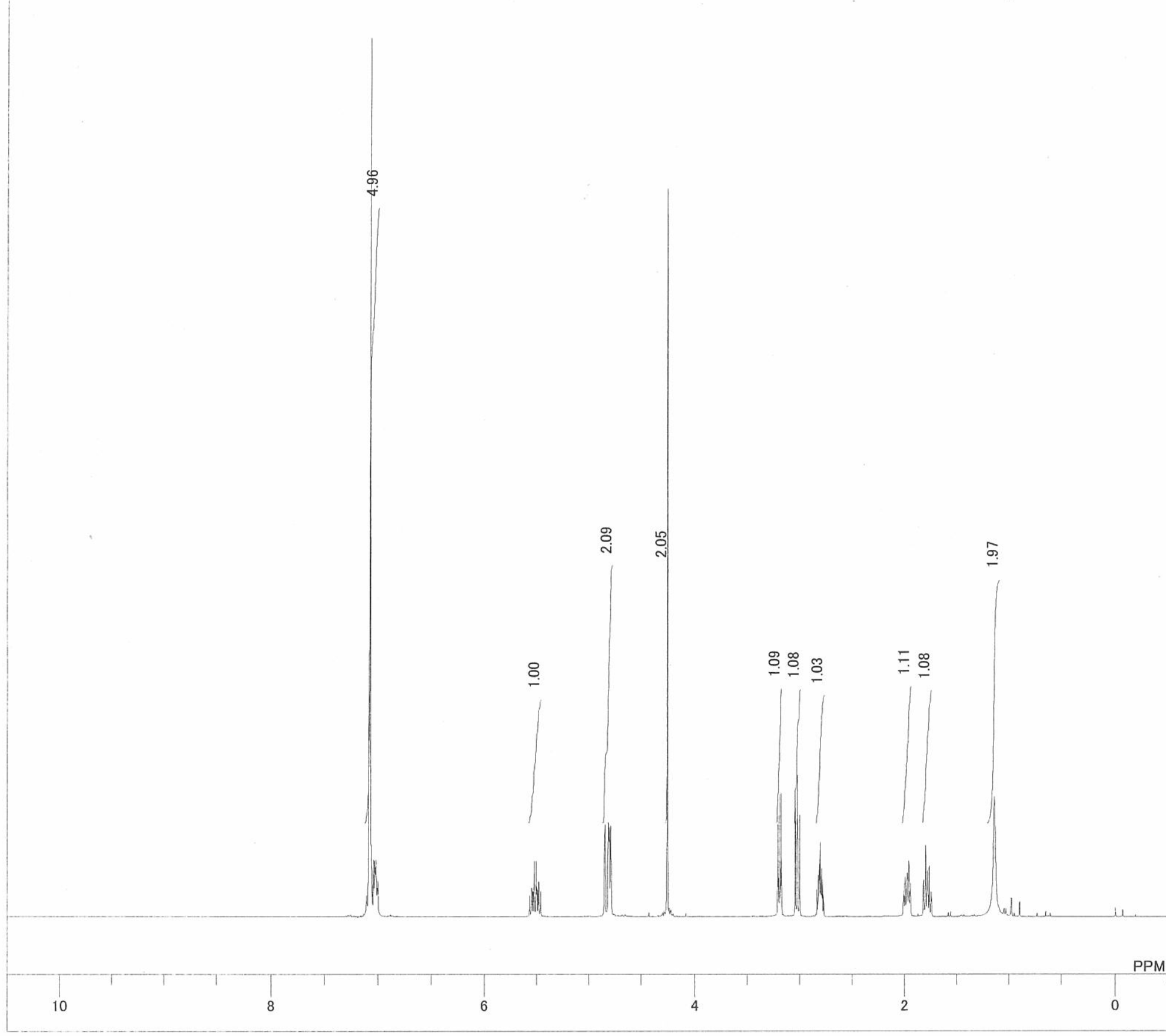

DATIM

EXMOD

EXMOD

OBFRQ

OBFIN

POINT

FREQU

SCANS

ACQTM

PD

PW1

RNNC

STNT

CLVT

BF

$\because$ Documents and Settings $¥$ delta $¥$ My Documents $¥$ m3-31-OH-pure

ingle_pulse.ex2

$399.78 \mathrm{MHz}$
$4.19 \mathrm{KHz}$

$4.19 \mathrm{KHz}$
$7.29 \mathrm{~Hz}$

13107

$6002.31 \mathrm{~Hz}$

8
$2.1837 \mathrm{sec}$

$2.0000 \mathrm{sec}$

5.50 usec

$22.2 \mathrm{c}$

(

$0.00 \mathrm{ppm}$

28

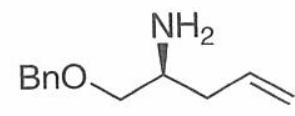

30 
DFILE

COMNT

DATIM

OBNUC

OXMOD

OBFRQ

OBSET

POINT

FREQU

SCANS

ACQTM

PD

PW1

IRNUC

CTEMP

SLVNT

EXREF

RGAIN

列

cm3-31-OH-pure-13C

130

single_pulse_dec

$$
\begin{gathered}
100.53 \mathrm{MHz} \\
5.35 \mathrm{KHz}
\end{gathered}
$$
$5.35 \mathrm{KHz}$
$5.86 \mathrm{~Hz}$ 32768 $31407.04 \mathrm{~Hz}$ 69 $1.0433 \mathrm{sec}$
$2.0000 \mathrm{sec}$ $\mathrm{H}$ 3.33 usec 22.6 $77.00 \mathrm{pp}$ $77.00 \mathrm{ppm}$
$0.12 \mathrm{~Hz}$

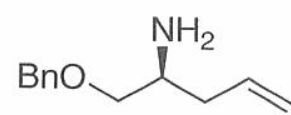

30

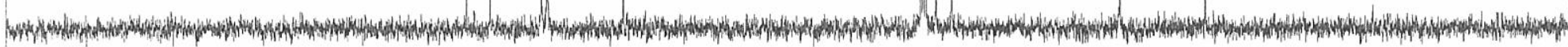


DFILE

COMNT

DATIM

OBNUC

EXMOD

OBFRQ

OBSET

OBFIN

PEOU

FREQU

SCANS

ADQT

PW1

IRNUC

CTEMP

LVNT

RGAIN

9-04-2006 10:04:2

single_pulse.ex2 $399.78 \mathrm{MHz}$ $4.19 \mathrm{KHz}$ $7.29 \mathrm{~Hz}$ (135)

$2.1837 \mathrm{sec}$

$2.0000 \mathrm{sec}$

5.50 usec

$\mathrm{CDCL}^{23.4 \mathrm{c}}$

$7.24 \mathrm{ppm}$

$0.12 \mathrm{~Hz}$

34

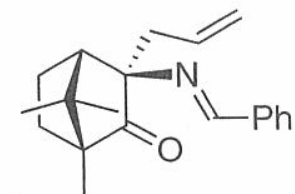

$4 b$ 
C: $¥$ Documents and Settings $¥$ delta $¥$ My Documents $¥$ Personal Folder $¥$ Morichie $¥ \mathrm{~cm} 3-60 ¥ \mathrm{~cm} 3-69-13 \mathrm{C}-1$.als cm3-69-13C-

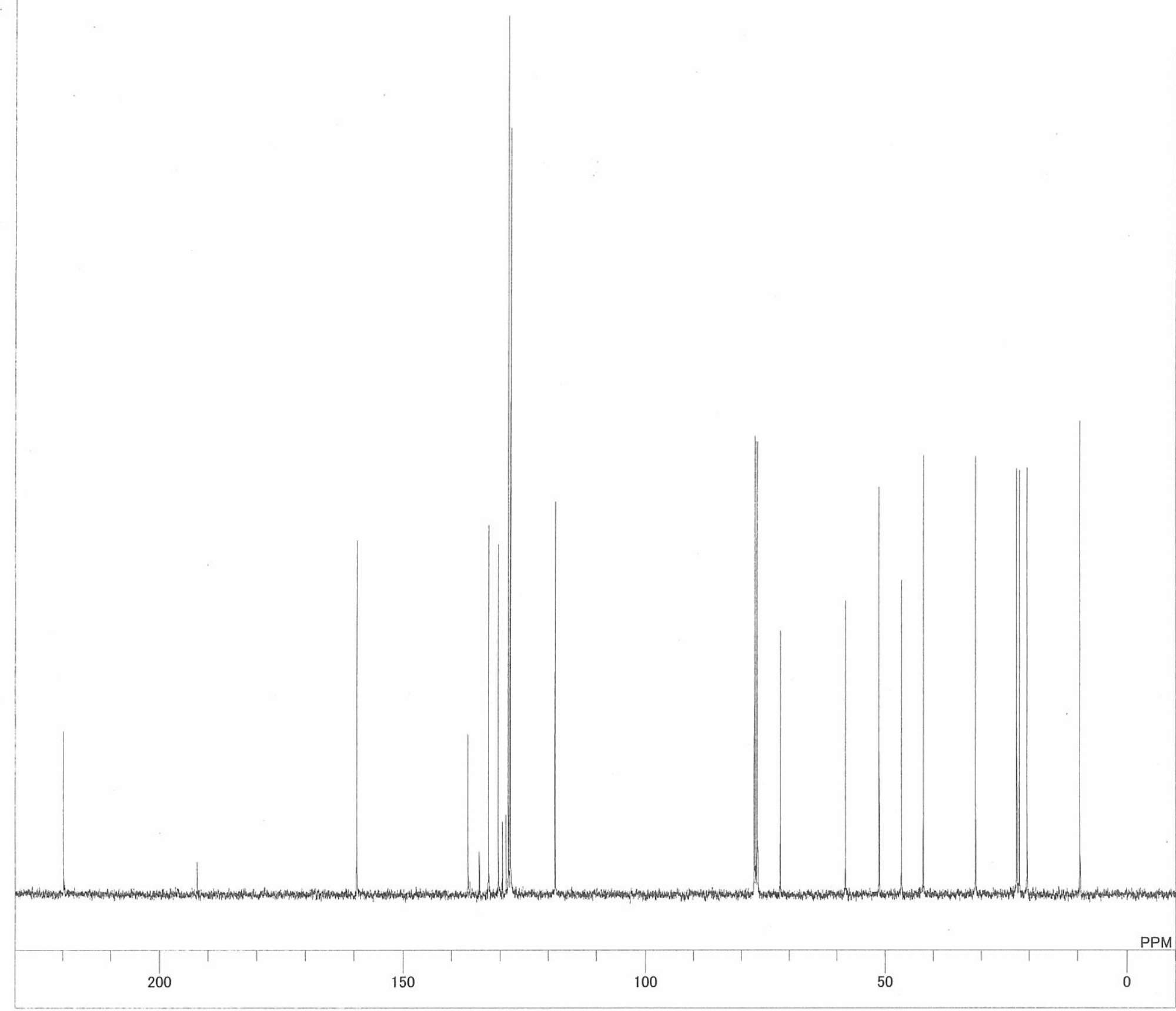

EXMOD

EXMUC

BXRQ

OBFIN

POINT

PEQU

SCANS

ACQTM

PW1

RNN

TLVMP

CXRF

RGAIN

:¥Documents and Settings $¥$ delta $¥ M y$ Documents $¥$

10:25:30

0. ingulse_dec
$100.53 \mathrm{MHz}$ $5.35 \mathrm{KHz}$ $5.86 \mathrm{~Hz}$ 32768 $1407.04 \mathrm{~Hz}$ 201

$1.0433 \mathrm{sec}$ $2.0000 \mathrm{sec}$
$3.33 \mathrm{usec}$ 23.9 c DCL 00 $77.00 \mathrm{ppm}$
$0.12 \mathrm{~Hz}$
50

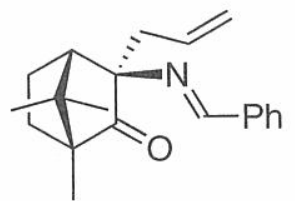

$4 b$ 
C:¥Documents and Settings $¥ A l l$ Users $¥$ Documents $¥$ Morichie $¥ \mathrm{~cm} 3-85$-down_PROTON-lit.als

cm3-85-down

DFILE

COMNT

DATIM

OBNUC

EXMOD

OBFRQ

OBSET

OBFIN

POINT

FREQU

SCANS

ACQTM

PD

PW1

IRNUC

CTEMP

SLVNT

EXREF

BF

RGAIN

C:¥Documents and Settings $¥$ All Users $¥$ cm3-85-down

2-05-2006 01:06:22

$1 \mathrm{H}$

single_pulse.ex2

$600.17 \mathrm{MHz}$

$5.30 \mathrm{KHz}$

$5.47 \mathrm{~Hz}$

13107

$9008.87 \mathrm{~Hz}$

16

$1.4549 \mathrm{sec}$

$4.0000 \mathrm{sec}$

$1 \mathrm{H}$

$20.3 \mathrm{c}$

$0.00 \mathrm{ppm}$

$0.12 \mathrm{~Hz}$

ญั

10

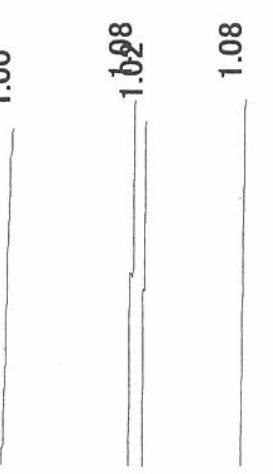

(n)

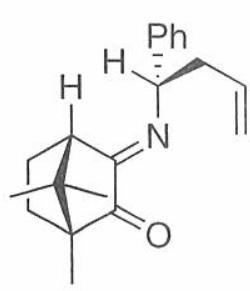

(E)-5b

$(95 \%$ E) 
C: ¥Documents and Settings¥All Users ¥Documents $¥$ Morichie $¥ \mathrm{~cm} 3-85$-down_CARBON-lit.als cm3-85-don

DFILE

COMNT

DATIM

OBNUC

EXMOD

OBFRQ

OBSET

OBFIN

POINT

FREQU

SCANS

ACQTM

PD

PD

IRNUC

CTEMP

SLVNT

EXREF

BF

RGAIN

3-85-down

-05-2006 03:45:38

single_pulse_dec

$150.92 \mathrm{MHz}$

$8.52 \mathrm{KHz}$

$1.74 \mathrm{H}$

32768

$47348.48 \mathrm{~Hz}$

1024

$0.6921 \mathrm{sec}$

$2.0000 \mathrm{sec}$

3.83 usec

20.6

$128.00 \mathrm{ppm}$ $0.12 \mathrm{~Hz}$

58

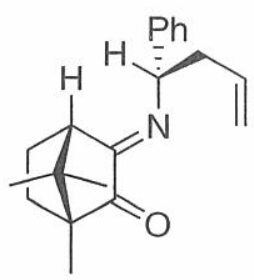

(E)-5b

(95\% E)

Why

PPM 
C: $¥ D o c u m e n t s$ and Settings $¥ A l l$ Users $¥ D o c u m e n t s ¥ M o r i c h i e ¥ c m 3-85-$ up_PROTON.als cm3-85-up

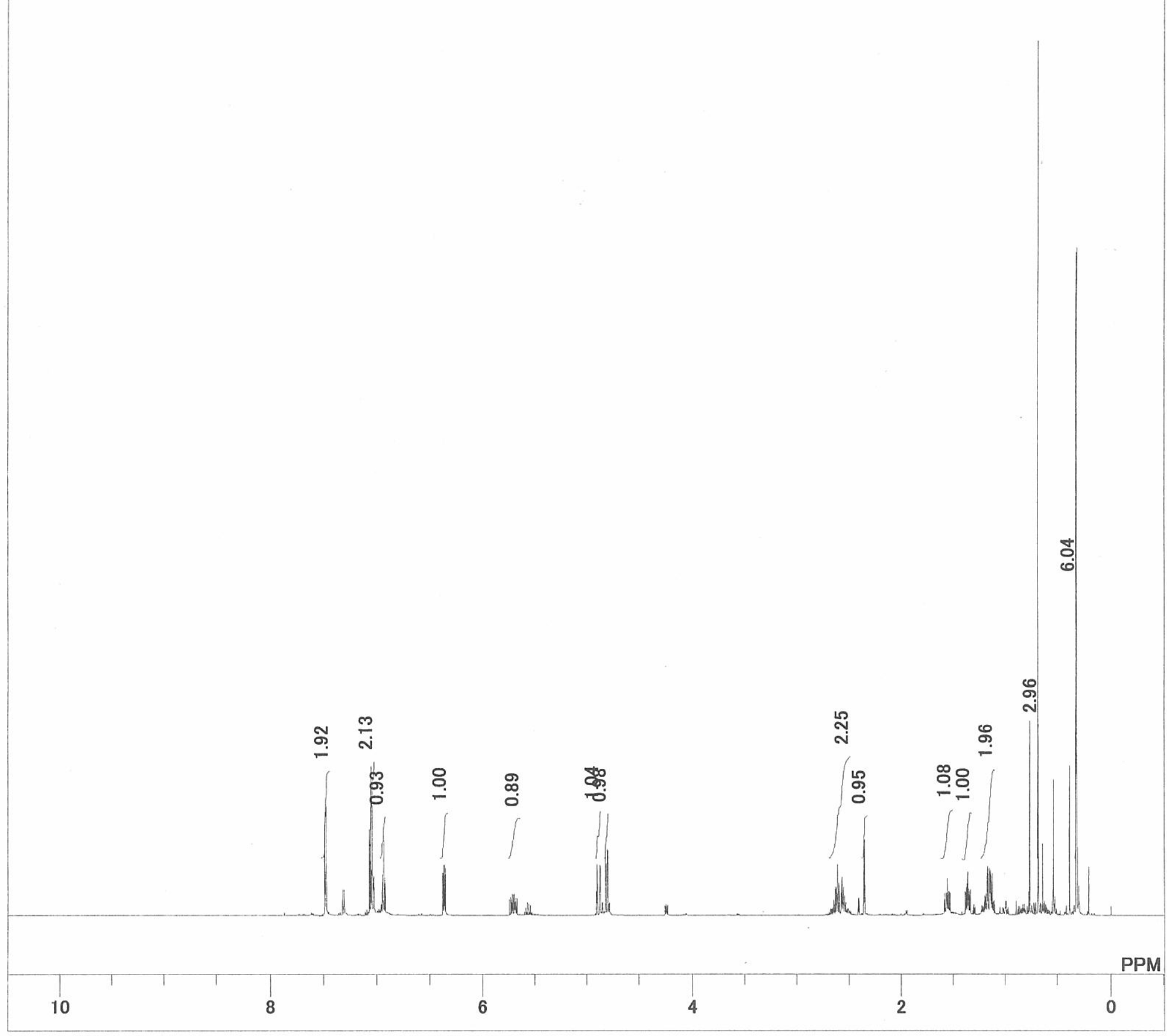

DFILE

COMNT

DATIM

EXMOD

EXMOD

OBFRQ

OBSE

OBFIN

POINT

FREQU

SCANS

ACQTM

PD

PW1

RNUC

CTEMP
SLVNT

EXREF

BF

RGAIN

C:¥Documents and Settings $¥$ All Users $¥$ cm3-85-up

01-05-2006 22:04:19

le_pulse.ex2

600.17 MH

$5.30 \mathrm{KHz}$

$5.47 \mathrm{~Hz}$

13107

$9008.87 \mathrm{~Hz}$

$1.4549 \mathrm{sec}$

4.0000 sec

7.30 usec

$1 \mathrm{H}$

7.30 usec

C6D 6

$20.4 c$

$0.00 \mathrm{ppm}$

$0.12 \mathrm{~Hz}$

40

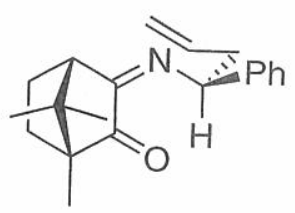

(Z) $-5 b$

$(80 \%$ Z) 
C:¥Documents and Settings $¥ A l l$ Users $¥ D o c u m e n t s ¥ M o r i c h i e ¥ c m 3-85-$ up_CARBON.als cm3-85-up

DFILE

COMNT

DATIM

OBNUC

EXMOD

OBFRQ

OBSET

SCANS

ACQTM

PD

PW1

IRNUC

CTEMP

SLVNT

EXRE

RGAIN
C:¥Documents and Settings $¥$ All Users cm3-85-up

02-05-2006 00:43:11

13C

single_pulse_dec

$50.92 \mathrm{MHz}$

$8.52 \mathrm{KHz}$

$1.74 \mathrm{~Hz}$

$47348.48 \mathrm{~Hz}$

1024

$0.6921 \mathrm{sec}$

$2.0000 \mathrm{sec}$

$1 \mathrm{H}$

83 usec

$21.0 \mathrm{c}$

$128.00 \mathrm{ppm}$

$0.12 \mathrm{~Hz}$

58

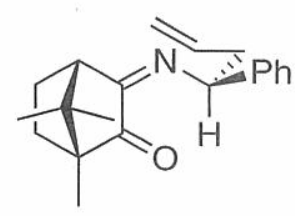

(Z) $-5 \mathrm{~b}$

$(80 \%$ Z) 